\title{
The Proper Dissipative Extensions of a Dual Pair
}

\author{
Christoph Fischbacher, Sergey Naboko and Ian Wood
}

\begin{abstract}
Let $A$ and $(-\widetilde{A})$ be dissipative operators on a Hilbert space $\mathcal{H}$ and let $(A, \widetilde{A})$ form a dual pair, i.e. $A \subset \widetilde{A}^{*}$, resp. $\widetilde{A} \subset A^{*}$. We present a method of determining the proper dissipative extensions $\widehat{A}$ of this dual pair, i.e. $A \subset \widehat{A} \subset \widetilde{A}^{*}$ provided that $\mathcal{D}(A) \cap \mathcal{D}(\widetilde{A})$ is dense in $\mathcal{H}$. Applications to symmetric operators, symmetric operators perturbed by a relatively bounded dissipative operator and more singular differential operators are discussed. Finally, we investigate the stability of the numerical range of the different dissipative extensions.
\end{abstract}

Mathematics Subject Classification (2010). Primary 47B44; Secondary 47A20.

Keywords. Dissipative Operators, Operator Extensions, Dual Pairs.

\section{Introduction}

The purpose of this paper is to develop a straightforward method for computing the proper dissipative extensions of a given dual pair of operators $(A, \widetilde{A})$, where $A$ and $(-\widetilde{A})$ are dissipative, under the mild assumption that $\mathcal{D}(A) \cap \mathcal{D}(\widetilde{A})$ is dense.

Numerous authors have contributed to the study of abstract extension problems for operators on Hilbert spaces, which goes at least back to von Neumann [38] whose well-known von Neumann formulas provide a full characterization of all selfadjoint extensions of a given symmetric operator. As it would be impossible to give an exhaustive overview here, let us just mention the results of Kreı̆n, Vishik, Birman and Grubb ([32], [42], [10] and [28]) who described all positive selfadjoint extensions of a given positive symmetric operator using positive selfadjoint operators on an auxiliary boundary space (cf. the survey [1] as well as the addendum acknowledging Grubb's contributions to the field [2]). Beyond that, Grubb's methods also allowed her to determine sectorial and $m$-sectorial extensions of positive symmetric operators [28]. For a much broader overview over the field, let us point the interested reader to the survey [8] and all the references therein (in particular also to the study of the 
extensions of linear relations rather than just operators).

In his seminal paper [39], Phillips coined the term of a dissipative operator. He showed that dissipative operators always allow for maximally dissipative extensions, which are generators of $C_{0}$-semigroups of contractions. In order to determine these maximally dissipative extensions, he employed Kreln space methods as well as finding contractive extensions of the Cayley transform associated to the operator.

Lyantze and Storozh determined the maximally dissipative extensions of operators that one obtains by slightly varying abstract boundary conditions in the domain of certain symmetric operators such that the resulting operators are dissipative [33].

Moreover, for the sectorial case and for contributions towards extensions of dual pairs of operators, authors like Arlinskiü, Derkach, Kovalev, Malamud, Mogilevskii and Tsekanovskiu $[4,6,7,16,35,36,37]$ have made many contributions using form methods and boundary triples in order to determine $m$-sectorial and $m$-accretive extensions (for an overview cf. [5] and all the references therein). In particular for boundary triples, there has recently been a significant increase of interest with special attention towards their applications to PDE problems usually in the selfadjoint case $[12,13,14,21,22$, 23, 24, 25, 26, 27, 29]. Let us also point out examples, where explicit computations of maximally dissipative (resp. accretive) extensions for positive symmetric differential operators [19], [20] and for sectorial Sturm-Liouville operators [11] have been performed. Lastly, let us also mention that some recent developments in the theory of maximal monotone nonlinear operators can be found in [41].

We will proceed as follows:

In Section 2, we will give a few basic definitions and recall some useful results regarding dual pairs and dissipative operators and their extensions.

In Section 3, we introduce the common core property of a dual pair $(A, \widetilde{A})$, which ensures that the dual pair under consideration provides us with a convenient way of defining an operator $V$ that corresponds to the "imaginary part" of $A$.

It will be the square root of the selfadjoint Krĕn-von Neumann extension of $V$ - denoted by $V_{K}^{1 / 2}$ - which will play an important role for the results obtained in Section 4. The description of $V_{K}^{1 / 2}$ obtained by Ando and Nishio [3] will allow us to give a necessary and sufficient condition (Theorem 4.7) for an extension of $(A, \widetilde{A})$ to be dissipative, which we only have to check on the space by which we extend the operator $A$ rather than on the whole domain of the extension. From this result, we proceed to give a description of all dissipative extensions of the dual pair $(A, \widetilde{A})$ in terms of contractions from one "small" auxiliary space to another. We also generalize our results to the case that the common core property is not satisfied by the dual pair as long as $\mathcal{D}(A) \cap \mathcal{D}(\widetilde{A})$ is still dense. As a first application, we start by considering symmetric operators with relatively bounded dissipative perturbations and 
after that, we consider more singular dissipative operators - our first examples being such that the associated imaginary part $V$ is already essentially selfadjoint and our last example being such that there is a family of selfadjoint extensions of $V$.

Finally, in Section 5, we find lower bounds for the numerical range of the dissipative extensions we have obtained and apply this result to the examples from the previous section.

\section{Acknowledgements}

It is a pleasure to acknowledge Malcolm Brown for useful discussions and pointing out numerous references. C.F. would also like to thank Petr Siegl for providing him with the reference for the Hess-Kato Theorem [30] and Eduard Tsekanovskil for communicating various references. C.F. acknowledges the UK Engineering and Physical Science Research Council (Doctoral Training Grant Ref. EP/K50306X/1) and the School of Mathematics, Statistics and Actuarial Science at the University of Kent for a PhD studentship. S.N. was supported by the Russian Science Foundation (grant number 15-11-30007). S.N. also expresses his deep gratitude to the University of Kent at Canterbury for the hospitality and Marie Curie grant (2013-2014), PIIF-GA-2011-299919. We are also grateful to the referee for helpful remarks and pointing out a shortcut to the proof of Theorem 4.7.

\section{Some definitions and conventions}

\subsection{Dissipative operators}

Let us begin with a few basic definitions and results on dissipative operators.

Definition 2.1. An operator $A$ on a Hilbert space $\mathcal{H}$ is said to be dissipative if and only if it is densely defined and

$$
\operatorname{Im}\langle f, A f\rangle \geq 0
$$

for all $f \in \mathcal{D}(A)$. An operator $\widetilde{A}$ is called antidissipative if and only if $(-\widetilde{A})$ is dissipative.

Note that we have defined the scalar product $\langle\cdot, \cdot\rangle$ to be antilinear in the first and linear in the second component. Also note that we require $A$ to be densely defined for it to be dissipative. Finally, let us remark that any operator $A$, which is dissipative in the above sense, is also closable with its closure $\bar{A}$ being dissipative as well [39].

Definition 2.2. A dissipative operator $A$ is said to be maximally dissipative if for any dissipative operator extension $A \subset A^{\prime}$ we get that $A=A^{\prime}$.

Let us remark at this point that the distinction between $m$-dissipative and maximally dissipative operators as it can be found in the literature (cf. 
e.g. [18, Sec. 3] for accretive operators) is not needed if one only considers densely defined dissipative operators as they coincide for this case. The following result is a well known fact:

Proposition 2.3 ([39, Theorems 1.1.1, 1.1.2 and 1.1.3]). Let $A$ be dissipative. Then, the following are equivalent:

- A is maximally dissipative.

- There exists a $\lambda \in \mathbb{C}$ with $\operatorname{Im}(\lambda)<0$ such that $\lambda \in \rho(A)$, where $\rho(A)$ denotes the resolvent set of $A$.

- $\mathbb{C}^{-}:=\{z \in \mathbb{C}: \operatorname{Im}(z)<0\} \subset \rho(A)$.

- $\left(-A^{*}\right)$ is dissipative.

- $i A$ is the generator of a strongly continuous semigroup of contractions on $\mathcal{H}$.

Finally, let us state a lemma on by how many linearly independent vectors the domain of a given closed dissipative operator with finite defect index has to increase in order to obtain a maximally dissipative extension.

Lemma 2.4 ([15]). Let $A$ be a closed and dissipative linear operator on a separable Hilbert space $\mathcal{H}$ such that $\operatorname{dim}[\operatorname{ran}(A+i)]^{\perp}<\infty$. Moreover, let $A^{\prime}$ be a dissipative extension of $A$. Then, $A^{\prime}$ is maximally dissipative if and only if

$$
\operatorname{dim} \mathcal{D}\left(A^{\prime}\right) / \mathcal{D}(A)=\operatorname{dim}[\operatorname{ran}(A+i)]^{\perp} .
$$

\subsection{Dual pairs}

Let us introduce the notion of a dual pair of operators (see also [33] for more details). Given a densely defined closable operator $A$, it is a well known fact that another densely defined closable operator $\widetilde{A}$ can always be found such that $(A, \widetilde{A})$ forms a dual pair as can be seen from the trivial choice $\widetilde{A}:=A^{*}$.

Definition 2.5. Let $(A, \widetilde{A})$ be a pair of densely defined and closable operators. We say that they form a dual pair if

$$
A \subset \widetilde{A}^{*} \text { resp. } \tilde{A} \subset A^{*} .
$$

In this case, $A$ is called a formal adjoint of $\widetilde{A}$ and vice versa.

Dual pairs can be thought of as a pair consisting of a "maximal" operator (in our notation $\widetilde{A}^{*}$ ) and a "minimal" operator (here: $A$ ). In this sense, any extension of $A$ that is a restriction of $\widetilde{A}^{*}$ can be interpreted as preserving the formal action of $\widetilde{A}^{*}$ :

Definition 2.6. Let $(A, \widetilde{A})$ be a dual pair. An operator $A^{\prime}$ is said to be a proper extension of the dual pair $(A, \widetilde{A})$ if

$$
A \subset A^{\prime} \subset \widetilde{A}^{*} \text { resp. } \widetilde{A} \subset\left(A^{\prime}\right)^{*} \subset A^{*} .
$$

Let us quote two useful results on the existence of proper extensions of certain dual pairs. The first proposition guarantees the existence of a proper extension of a dual pair $(A, \widetilde{A})$ with $\lambda \in \widehat{\rho}(A)$ and $\bar{\lambda} \in \widehat{\rho}(\widetilde{A})$, where $\widehat{\rho}(A)$ 
denotes the field of regularity of the operator $A$ (for a definition see e.g. [43]). This applies in particular if $A$ is dissipative, which means that $\mathbb{C}^{-} \subset \widehat{\rho}(A)$ and if $\widetilde{A}$ is antidissipative, which implies $\mathbb{C}^{+} \subset \widehat{\rho}(\widetilde{A})$.

Proposition 2.7 ([28, Chapter II, Lemma 1.1]). Let $(A, \widetilde{A})$ be a dual pair with $\lambda \in \widehat{\rho}(A)$ and $\bar{\lambda} \in \widehat{\rho}(\widetilde{A})$. Then there exists a proper extension $\widehat{A}$ of $(A, \widetilde{A})$ such that $\lambda \in \rho(\widehat{A})$ and $\mathcal{D}\left(\widetilde{A}^{*}\right)$ can be expressed as

$$
\mathcal{D}\left(\widetilde{A}^{*}\right)=\mathcal{D}(\bar{A}) \dot{+}(\widehat{A}-\lambda)^{-1} \operatorname{ker}\left(A^{*}-\bar{\lambda}\right) \dot{+} \operatorname{ker}\left(\widetilde{A}^{*}-\lambda\right) .
$$

Likewise, we get the following description for $\mathcal{D}\left(A^{*}\right)$ :

$$
\mathcal{D}\left(A^{*}\right)=\mathcal{D}(\overline{\widetilde{A}}) \dot{+}\left(\widehat{A}^{*}-\bar{\lambda}\right)^{-1} \operatorname{ker}\left(\widetilde{A}^{*}-\lambda\right) \dot{+} \operatorname{ker}\left(A^{*}-\bar{\lambda}\right) .
$$

The following proposition guarantuees the existence of a proper maximally dissipative extension for any dual pair $(A, \widetilde{A})$, where $A$ is dissipative and $\widetilde{A}$ is antidissipative. Up to a suitable multiplication by $i$, a proof for this can be found in [40, Chapter IV, Proposition 4.2].

Proposition 2.8. Let $(A, \widetilde{A})$ be a dual pair, where $A$ is dissipative and $\widetilde{A}$ is antidissipative. Then there exists a maximally dissipative proper extension of $(A, \widetilde{A})$.

Finally, let us introduce some convenient notation for complementary subspaces:

Definition 2.9. Let $\mathcal{N}$ be a (not necessarily closed) linear space and $\mathcal{M} \subset \mathcal{N}$ be a (not necessarily closed) subspace. With the notation $\mathcal{N} / / \mathcal{M}$ we mean any subspace of $\mathcal{N}$, which is complementary to $\mathcal{M}$, i.e.

$$
(\mathcal{N} / / \mathcal{M})+\mathcal{M}=\mathcal{N} \quad \text { and } \quad(\mathcal{N} / / \mathcal{M}) \cap \mathcal{M}=\{0\} .
$$

\section{The common core property}

In many situations (including all of the examples that we are going to discuss in this paper) one considers dual pairs of operators, which are constructed by firstly defining them on a common core like, e.g. the compactly supported smooth functions, and then taking closures:

Definition 3.1. Let $(A, \widetilde{A})$ be a dual pair of closed operators. We say that it has the common core property if $\overline{A \uparrow_{\mathcal{D}(A) \cap \mathcal{D}(\widetilde{A})}}=A$ and $\widetilde{A}=\overline{\widetilde{A} \uparrow_{\mathcal{D}(A) \cap \mathcal{D}(\widetilde{A})}}$.

Example 3.2. Consider the dissipative momentum operator $T$ given by

$$
T: \quad \mathcal{D}(T)=\left\{f \in H^{1}(0,1), f(0)=\rho f(1)\right\}, \quad f \mapsto i f^{\prime},
$$

where $|\rho|<1$. Here, $f^{\prime}$ denotes the weak derivative of $f$. Its adjoint $T^{*}$ is given by

$$
T^{*}: \quad \mathcal{D}\left(T^{*}\right)=\left\{f \in H^{1}(0,1), \bar{\rho} f(0)=f(1)\right\}, \quad f \mapsto i f^{\prime} .
$$

Clearly, $\left(T, T^{*}\right)$ is a dual pair. However, since $\mathcal{D}:=\mathcal{D}(T) \cap \mathcal{D}\left(T^{*}\right)=\{f \in$ $\left.H^{1}(0,1), f(0)=f(1)=0\right\}$, this dual pair does not have the common core 
property, as $S:=\overline{T\lceil\mathcal{D}}$ is symmetric and a proper restriction of $T$.

More generally, let $S$ be a closed and symmetric (in particular densely defined) operator. Moreover, let $S^{\prime}$ be any closed (not necessarily symmetric) extension of $S$ such that $S \subset S^{\prime} \subset S^{*}$. This readily implies that $\left(S, S^{\prime}\right)$ is a dual pair. However, since $\mathcal{D}(S) \cap \mathcal{D}\left(S^{\prime}\right)=\mathcal{D}(S)$, we get $S=\overline{S^{\prime}\left\lceil\mathcal{D}(S) \cap \mathcal{D}\left(S^{\prime}\right)\right.}$. Thus, the only dual pair of this form, which has the common core property is $(S, S)$. Moreover, let $V \geq 0$ be $S^{*}$-bounded with $S^{*}$-bound less than 1, which implies in particular that $V$ is $S^{\prime}$-bounded with $S^{\prime}$-bound less than 1 (for a definition of relative boundedness, see e.g. [31]). By the Hess-Kato Theorem $\left[30\right.$, Corollary 1], we have that $\left(S^{\prime}+i V\right)^{*}=S^{\prime *}-i V \subset S^{*}-i V$. This implies again that any pair of the form $\left(S+i V, S^{\prime}-i V\right)$ is a dual pair. However, again we have that the only dual pair which has the common core property is $(S+i V, S-i V)$.

The following lemma shows in particular that if we have a dual pair $(A, \widetilde{A})$ that has the common core property, where $A$ is dissipative, one can conclude that $\widetilde{A}$ is antidissipative.

Lemma 3.3. Let $(A, \widetilde{A})$ be a dual pair of closed operators, which has the common core property. Moreover, let $\mathcal{N}_{A}:=\{\langle f, A f\rangle: f \in \mathcal{D}(A),\|f\|=1\}$ be the numerical range of $A$ and let $\mathcal{N}_{\widetilde{A}}^{*}:=\{\overline{\langle f, \widetilde{A} f\rangle}: f \in \mathcal{D}(\widetilde{A}),\|f\|=1\}$ be the complex conjugate of the numerical range of $\widetilde{A}$. Then, the closures of the numerical range of $A$ and the complex conjugate of the numerical range of $\widetilde{A}$ coincide:

$$
\overline{\mathcal{N}_{A}}=\overline{\mathcal{N}_{\widetilde{A}}^{*}} \text {. }
$$

Proof. Let $f \in \mathcal{D}(A)$ be normalized. Since $\mathcal{D}(A) \cap \mathcal{D}(\widetilde{A})$ is a core for $A$, there exists a normalized sequence $\left\{f_{n}\right\}_{n} \subset \mathcal{D}(A) \cap \mathcal{D}(\widetilde{A})$ such that $f_{n} \rightarrow f$ and $A f_{n} \rightarrow A f$ for $n \rightarrow \infty$. Using that $\left\langle f_{n}, A f_{n}\right\rangle=\overline{\left\langle f_{n}, \widetilde{A} f_{n}\right\rangle}$, we get that

$$
\lim _{n \rightarrow \infty} \overline{\left\langle f_{n}, \widetilde{A} f_{n}\right\rangle}=\lim _{n \rightarrow \infty}\left\langle f_{n}, A f_{n}\right\rangle=\langle f, A f\rangle .
$$

Since $\left\{\overline{\left\langle f_{n}, \widetilde{A} f_{n}\right\rangle}\right\}_{n}$ is a sequence of elements in $\mathcal{N}_{\widetilde{A}}^{*}$, we get that $\langle f, A f\rangle$ is a limit point of $\mathcal{N}_{\widetilde{A}}^{*}$, which means that

$$
\mathcal{N}_{A} \subset \overline{\mathcal{N}_{\widetilde{A}}^{*}} .
$$

By similar reasoning, we get that

$$
\mathcal{N}_{\widetilde{A}}^{*} \subset \overline{\mathcal{N}_{A}}
$$

which - after taking closures - yields the lemma.

Remark 3.4. If $A$ is closed and dissipative and $\mathcal{D}(A) \cap \mathcal{D}\left(A^{*}\right)$ is a core for $A$, i.e. $A=\overline{A\left\lceil\mathcal{D}(A) \cap \mathcal{D}\left(A^{*}\right)\right.}$, we can define $\widetilde{A}:=\overline{A^{*}\left\lceil\mathcal{D}(A) \cap \mathcal{D}\left(A^{*}\right)\right.}$, to construct a dual pair $(A, \widetilde{A})$, which has the common core property. This is in particular possible for the case that $\mathcal{D}(A) \subset \mathcal{D}\left(A^{*}\right)$ (cf. [40, Corollary to Proposition IV, 4.2$])$. 


\section{The main theorem}

In this section, we will prove the main result, which can be written in a particularly nice form, if the common core property is satisfied. As any dissipative operator is closable with its closure being dissipative as well, it is necessary and sufficient to check dissipativity of an operator restricted to a core.

Lemma 4.1. Let $A$ be a closed, densely defined operator and let $\mathcal{C} \subset \mathcal{H}$ be a core for $A$. Moreover, assume that $B$ is a closed extension of $A$, i.e. $A \subset B$ and $\mathcal{D}(B)=\mathcal{D}(A) \dot{+} \mathcal{M}$. Then, $\mathcal{C}+\mathcal{M}$ is a core for $B$.

Proof. Since $\mathcal{C}$ is a core for $A$, this means that for every $f \in \mathcal{D}(A)$ there exists a sequence $\left\{f_{n}\right\}_{n} \subset \mathcal{C}$ such that $f_{n} \rightarrow f$ and $A f_{n} \rightarrow A f$ and therefore for any element of $\mathcal{D}(B) \ni(f+m)$, where $f \in \mathcal{D}(A)$ and $m \in \mathcal{M}$ we get

$\left(f_{n}+m\right) \rightarrow(f+m)$ and $B\left(f_{n}+m\right)=\left(A f_{n}+B m\right) \rightarrow(A f+B m)=B(f+m)$, which is the desired result.

For the following results, let us recall the definition of the Krel̆n-von Neumann extension of a symmetric non-negative operator:

Definition 4.2. Let $V$ be symmetric and non-negative operator, i.e. $\langle f, V f\rangle \geq$ 0 for all $f \in \mathcal{D}(V)$. Then, the Kreĭn-von Neumann extension of $V$, which we denote by $V_{K}$, is the smallest non-negative selfadjoint extension of $V$, i.e. for any $\widehat{V}=\widehat{V}^{*}$ with $V \subset \widehat{V}$ and $\widehat{V} \geq 0$ we have that

$$
0 \leq V_{K} \leq \widehat{V} \text {. }
$$

It is a well known fact that such an extension $V_{K}$ always exists and that it is unique (cf. [32]).

(Recall that for two non-negative selfadjoint operators $A$ and $B$ on a Hilbert space $\mathcal{H}$, the relation $A \leq B$ is defined as

$$
A \leq B: \Leftrightarrow \mathcal{D}\left(A^{1 / 2}\right) \supset \mathcal{D}\left(B^{1 / 2}\right) \text { and }\left\|A^{1 / 2} f\right\| \leq\left\|B^{1 / 2} f\right\|
$$

for all $f \in \mathcal{D}\left(B^{1 / 2}\right)$.)

For the special case that $V$ is strictly positive, i.e. there exists an $\varepsilon>0$ such $\langle f, V f\rangle \geq \varepsilon\|f\|^{2}$ for all $f \in \mathcal{D}(V)$, we have the following characterization of $V_{K}[1]$ :

$$
V_{K}: \quad \mathcal{D}\left(V_{K}\right)=\mathcal{D}(V) \dot{+} \operatorname{ker} V^{*}, \quad V_{K}=V^{*} \Gamma_{\mathcal{D}}\left(V_{K}\right)
$$

and for $V_{K}^{1 / 2}$ we get

$$
\begin{array}{cc}
V_{K}^{1 / 2}: & \mathcal{D}\left(V_{K}^{1 / 2}\right)=\mathcal{D}\left(V_{F}^{1 / 2}\right) \dot{+} \operatorname{ker} V^{*} \\
\left\langle V_{K}^{1 / 2}(f+k), V_{K}^{1 / 2}(f+k)\right\rangle=\left\langle V_{F}^{1 / 2} f, V_{F}^{1 / 2} f\right\rangle,
\end{array}
$$

with $f \in \mathcal{D}\left(V_{F}^{1 / 2}\right)$, where $V_{F}$ is the Friedrichs extension of $V$ and $k \in \operatorname{ker} V^{*}$.

For the proof of the main theorem without having to assume that the imaginary part is strictly positive, we will make use of an equivalent description for non-negative $V_{K}^{1 / 2}$ proved by Ando and Nishio. 
Proposition 4.3 (T. Ando, K. Nishio, [3, Thm. 1]). Let $V$ be a non-negative closed symmetric operator. The selfadjoint and non-negative square-root of the Krĕ̌n-von Neumann extension of $V$, which we denote by $V_{K}^{1 / 2}$ can be characterized as follows:

$$
\mathcal{D}\left(V_{K}^{1 / 2}\right)=\left\{h \in \mathcal{H}: \sup _{f \in \mathcal{D}(V): V f \neq 0} \frac{|\langle h, V f\rangle|^{2}}{\langle f, V f\rangle}<\infty\right\},
$$

for any $h \in \mathcal{D}\left(V_{K}^{1 / 2}\right): \quad\left\|V_{K}^{1 / 2} h\right\|^{2}=\sup _{f \in \mathcal{D}(V): V f \neq 0} \frac{|\langle h, V f\rangle|^{2}}{\langle f, V f\rangle}$.

Remark 4.4. We draw the reader's attention to a slight difference in the way, Proposition 4.3 was stated in [3], where the supremum is taken over all $f \in \mathcal{D}(V)$ (without the extra condition that $V f \neq 0$ ), which only makes sense if one assumes that $\operatorname{ker} V=\{0\}$. The extra condition $V f \neq 0$ is a remedy for this problem and is a direct result from the reasoning of [3].

For our main theorem, we will make use of the fact that the dual pair under consideration has a common core $\mathcal{D}$, allowing us to define an "imaginary part" on $\mathcal{D}$. It will therefore be helpful to show that the supremum in Proposition 4.3 has to be taken only over $\mathcal{D}$.

Lemma 4.5. Let $V$ be a non-negative closed symmetric operator and $\mathcal{C}$ be a core for $V$. Then, for any $h \in \mathcal{H}$ we have that

$$
\sup _{f \in \mathcal{D}(V): V f \neq 0} \frac{|\langle h, V f\rangle|^{2}}{\langle f, V f\rangle}=\sup _{f \in \mathcal{C}: V f \neq 0} \frac{|\langle h, V f\rangle|^{2}}{\langle f, V f\rangle} .
$$

Proof. Let $s \in \mathbb{R}^{+} \cup\{\infty\}$ be defined as

$$
s:=\sup _{f \in \mathcal{D}(V): V f \neq 0} \frac{|\langle h, V f\rangle|^{2}}{\langle f, V f\rangle} .
$$

This means that there exists a sequence $\left\{f_{n}\right\}_{n} \subset \mathcal{D}(V)$ with $V f_{n} \neq 0$ such that

$$
\lim _{n \rightarrow \infty} \frac{\left|\left\langle h, V f_{n}\right\rangle\right|^{2}}{\left\langle f_{n}, V f_{n}\right\rangle}=s
$$

On the other hand, since $\mathcal{C}$ is a core for $V$, for any $f_{n} \in \mathcal{D}(V)$, there exists a sequence $\left\{f_{n, m}\right\}_{m} \subset \mathcal{C}$ such that

$$
\lim _{m \rightarrow \infty} f_{n, m}=f_{n} \quad \text { and } \quad \lim _{m \rightarrow \infty} V f_{n, m}=V f_{n} .
$$

Thus, for any fixed $h \in \mathcal{H}$ and $f_{n} \in \mathcal{D}(V)$ such that $V f_{n} \neq 0$, we have also $\left\langle f_{n}, V f_{n}\right\rangle \neq 0$ and therefore

$$
\lim _{m \rightarrow \infty} \frac{\left|\left\langle h, V f_{n, m}\right\rangle\right|}{\left\langle f_{n, m}, V f_{n, m}\right\rangle}=\frac{\left|\left\langle h, V f_{n}\right\rangle\right|}{\left\langle f_{n}, V f_{n}\right\rangle} .
$$

Hence, a diagonal sequence argument yields the lemma. 
Definition 4.6. Let $\mathcal{V} \subset \mathcal{D}\left(\widetilde{A}^{*}\right) / / \mathcal{D}(A)$ be a subspace. Then, the operator $A_{\mathcal{V}}$ is defined as

$$
A_{\mathcal{V}}: \quad \mathcal{D}\left(A_{\mathcal{V}}\right)=\mathcal{D}(A) \dot{+} \mathcal{V}, \quad A_{\mathcal{V}}=\widetilde{A}^{*}\left\lceil_{\mathcal{D}}\left(A_{\mathcal{V}}\right)\right.
$$

Theorem 4.7. Let $(A, \widetilde{A})$ be a dual pair of operators having the common core property, where $A$ is dissipative and let $\mathcal{D} \subset(\mathcal{D}(A) \cap \mathcal{D}(\widetilde{A}))$ be a common core for $A$ and for $\widetilde{A}$. Then, the operator $V:=\frac{A-\widetilde{A}}{2 i}$ defined on $\mathcal{D}$ is a nonnegative symmetric operator. Moreover, let $\mathcal{V} \subset \mathcal{D}\left(\widetilde{A}^{*}\right) / / \mathcal{D}(A)$ be a linear space. Then, the operator $A_{\mathcal{V}}$ is dissipative if and only if $\mathcal{V} \subset \mathcal{D}\left(V_{K}^{1 / 2}\right)$ and

$$
\operatorname{Im}\left\langle v, \widetilde{A}^{*} v\right\rangle \geq\left\|V_{K}^{1 / 2} v\right\|^{2} \quad \text { for all } v \in \mathcal{V} .
$$

The operator $V_{K}$ does not depend on the specific choice of $\mathcal{D}$ as long as $\mathcal{D} \subset(\mathcal{D}(A) \cap \mathcal{D}(\widetilde{A}))$ is a common core for $A$ and $\widetilde{A}$.

Proof. Since $\operatorname{Im}\langle f, A f\rangle \geq 0$ for all $f \in \mathcal{D}(A)$, by Lemma 3.3, this implies that $\operatorname{Im}\langle f, \widetilde{A} f\rangle \leq 0$ for all $f \in \mathcal{D}(\widetilde{A})$ and hence, $\widetilde{A}$ is anti-dissipative. Next, let us show that $V$ is symmetric and non-negative. For any $f \in \mathcal{D}$ we get

$$
\langle f, V f\rangle=\frac{1}{2 i}(\langle f, A f\rangle-\langle f, \widetilde{A} f\rangle)=\frac{1}{2 i}(\langle f, A f\rangle-\langle A f, f\rangle)=\operatorname{Im}\langle f, A f\rangle \geq 0
$$

by assumption. Let us now prove the criterion for dissipativity. By Lemma 4.1, it is sufficient to check dissipativity for all elements of $\mathcal{D}\left(A_{\mathcal{V}}\right)$, which are of the form $f+v$, where $f \in \mathcal{D}$ and $v \in \mathcal{V}$. Thus, it suffices to show that

$$
\operatorname{Im}\left\langle f+v, \widetilde{A}^{*}(f+v)\right\rangle \geq 0 \text { for all } f \in \mathcal{D}, v \in \mathcal{V}
$$

if $\mathcal{V} \subset \mathcal{D}\left(V_{K}^{1 / 2}\right)$ and $\operatorname{Im}\left\langle v, \widetilde{A}^{*} v\right\rangle \geq\left\|V_{K}^{1 / 2} v\right\|^{2}$ for all $v \in \mathcal{V}$. Then by (4.2):

$$
\begin{aligned}
\operatorname{Im}\left\langle f+v, \widetilde{A}^{*}(f+v)\right\rangle & =\operatorname{Im}\langle f, A f\rangle+\operatorname{Im}\left\langle v, \widetilde{A}^{*} v\right\rangle+\operatorname{Im}\left(\left\langle f, \widetilde{A}^{*} v\right\rangle+\langle v, A f\rangle\right) \\
& =\langle f, V f\rangle+\operatorname{Im}\left\langle v, \widetilde{A}^{*} v\right\rangle-\operatorname{Im}\langle(A-\widetilde{A}) f, v\rangle \\
& =\langle f, V f\rangle+\operatorname{Im}\left\langle v, \widetilde{A}^{*} v\right\rangle-\operatorname{Im}\langle 2 i V f, v\rangle .
\end{aligned}
$$

Observe that for any given $v$, one can always consider $e^{i \vartheta} v$ instead of $v$, where $\vartheta \in[0,2 \pi)$ is chosen such that $\operatorname{Im}\left\langle 2 i V f, e^{i \vartheta} v\right\rangle=-2|\langle V f, v\rangle|$ without changing the other two terms, which means that showing

$$
\langle f, V f\rangle+\operatorname{Im}\left\langle v, \widetilde{A}^{*} v\right\rangle-2|\langle V f, v\rangle| \geq 0 \text { for all } f \in \mathcal{D}, v \in \mathcal{V}
$$

is necessary and sufficient for $A_{\mathcal{V}}$ being dissipative.

Let us begin by showing that $\mathcal{V} \subset \mathcal{D}\left(V_{K}^{1 / 2}\right)$ and $\operatorname{Im}\left\langle v, \widetilde{A}^{*} v\right\rangle \geq\left\|V_{K}^{1 / 2} v\right\|^{2}$ is sufficient for $A_{\mathcal{V}}$ to be dissipative. Thus, let us now assume that these two assumptions are satisfied. Since $V \subset \bar{V} \subset V_{K}$ and $\mathcal{D}(V) \subset \mathcal{D}\left(V_{K}\right) \subset \mathcal{D}\left(V_{K}^{1 / 2}\right)$, this means that we can write $V f=V_{K} f=\left(V_{K}^{1 / 2}\right)\left(V_{K}^{1 / 2} f\right)$. We therefore 
get that

$$
\begin{aligned}
& \langle f, V f\rangle+\operatorname{Im}\left\langle v, \widetilde{A}^{*} v\right\rangle-2|\langle V f, v\rangle| \\
& \quad=\left\|V_{K}^{1 / 2} f\right\|^{2}+\operatorname{Im}\left\langle v, \widetilde{A}^{*} v\right\rangle-2\left|\left\langle V_{K}^{1 / 2} f, V_{K}^{1 / 2} v\right\rangle\right| \\
& \quad \geq\left\|V_{K}^{1 / 2} f\right\|^{2}+\operatorname{Im}\left\langle v, \widetilde{A}^{*} v\right\rangle-2\left\|V_{K}^{1 / 2} f\right\|\left\|V_{K}^{1 / 2} v\right\| \\
& \quad \geq\left\|V_{K}^{1 / 2} f\right\|^{2}+\left\|V_{K}^{1 / 2} v\right\|^{2}-2\left\|V_{K}^{1 / 2} f\right\|\left\|V_{K}^{1 / 2} v\right\| \\
& \quad=\left(\left\|V_{K}^{1 / 2} f\right\|-\left\|V_{K}^{1 / 2} v\right\|\right)^{2} \geq 0,
\end{aligned}
$$

Now, let us show that $A_{\mathcal{V}}$ being dissipative implies that $\mathcal{V} \subset \mathcal{D}\left(V_{K}^{1 / 2}\right)$ and $\operatorname{Im}\left\langle v, \widetilde{A}^{*} v\right\rangle \geq\left\|V_{K}^{1 / 2} v\right\|^{2}$ for all $v \in \mathcal{V}$. If $A_{\mathcal{V}}$ is dissipative, we get from (4.3) that for any $v \in \mathcal{V}$, we have that

$$
t^{2}\langle f, V f\rangle-2|t||\langle V f, v\rangle|+\operatorname{Im}\left\langle v, \widetilde{A}^{*} v\right\rangle \geq 0
$$

for all $t \in \mathbb{R}$ and all $f \in \mathcal{D}$, which implies that

$$
\frac{|\langle V f, v\rangle|^{2}}{\langle f, V f\rangle} \leq \operatorname{Im}\left\langle v, \widetilde{A}^{*} v\right\rangle
$$

for all $f \in \mathcal{D}$. From this, it follows that the supremum of the right hand side of (4.4) over all $f \in \mathcal{D}$ is bounded by $\operatorname{Im}\left\langle v, \widetilde{A}^{*} v\right\rangle$, which by Proposition 4.3 and Lemma 4.5 implies that $v \in \mathcal{D}\left(V_{K}^{1 / 2}\right)$ and $\operatorname{Im}\left\langle v, \widetilde{A}^{*} v\right\rangle \geq\left\|V_{K}^{1 / 2} v\right\|^{2}$.

Finally, let us show that for $\mathcal{D}^{\prime} \subset \mathcal{D}:=(\mathcal{D}(A) \cap \mathcal{D}(\widetilde{A}))$ both being common cores for $A$ and $\widetilde{A}$, we have that the Krein-von Neumann extensions of $V_{\mathcal{D}^{\prime}}=(A-\widetilde{A}) /(2 i) \Gamma_{\mathcal{D}^{\prime}}$ and $V_{\mathcal{D}}=(A-\widetilde{A}) /(2 i) \Gamma_{\mathcal{D}}$ coincide. As we have already shown that $V_{\mathcal{D}^{\prime}}$ and $V_{\mathcal{D}}$ are symmetric, it suffices to show that $\overline{V_{\mathcal{D}^{\prime}}}=\overline{V_{\mathcal{D}}}$. Since $V_{\mathcal{D}^{\prime}} \subset V_{\mathcal{D}}$, this will follow from $V_{\mathcal{D}} \subset \overline{V_{\mathcal{D}^{\prime}}}$. Using that $\mathcal{D}^{\prime}$ and $\mathcal{D}$ are cores for both $A$ and $\widetilde{A}$, we know that for any $f \in \mathcal{D}$, there exists a sequence $\left\{f_{n}\right\}_{n} \subset \mathcal{D}^{\prime}$ such that $f_{n} \rightarrow f$ and $A f_{n} \rightarrow A f$. Moreover, since $f \in \mathcal{D}(\widetilde{A})$ and $\mathcal{D}^{\prime}$ is a core for $\widetilde{A}$, this means that the sequence $\widetilde{A} f_{n}$ converges to $\widetilde{A} f$. Thus, for any $f \in \mathcal{D}$, there exists a sequence $\left\{f_{n}\right\}_{n} \subset \mathcal{D}^{\prime}$ such that $V_{\mathcal{D}^{\prime}} f_{n}=(2 i)^{-1}(A-\widetilde{A}) f_{n} \rightarrow(2 i)^{-1}(A-\widetilde{A}) f=V_{\mathcal{D}} f$, which implies that $V_{\mathcal{D}} \subset \overline{V_{\mathcal{D}^{\prime}}}$.

Corollary 4.8. Let $(A, \widetilde{A})$ be a dual pair satisfying the assumptions of Theorem 4.7. If for some $\lambda \in \mathbb{C}^{-}$we have that

$$
\operatorname{ker}\left(\widetilde{A}^{*}-\lambda\right) \cap \mathcal{D}\left(V_{K}^{1 / 2}\right)=\{0\},
$$

then there exists exactly one proper maximally dissipative extension of the dual pair $(A, \widetilde{A})$.

Proof. By Proposition 2.8, we know that there exists a maximally dissipative extension $\widehat{A}$ and by Proposition 2.3 , we know that $\mathbb{C}^{-} \in \rho(\widehat{A})$. Moreover, by [28] we have that

$$
\mathcal{D}(\widehat{A})=\mathcal{D}(A) \dot{+}(\widehat{A}-\lambda)^{-1} \operatorname{ker}\left(A^{*}-\bar{\lambda}\right)
$$


as well as

$$
\mathcal{D}\left(\widetilde{A}^{*}\right)=\mathcal{D}(A) \dot{+}(\widehat{A}-\lambda)^{-1} \operatorname{ker}\left(A^{*}-\bar{\lambda}\right) \dot{+} \operatorname{ker}\left(\widetilde{A}^{*}-\lambda\right) .
$$

By Theorem 4.7, we know that $(\widehat{A}-\lambda)^{-1} \operatorname{ker}\left(A^{*}-\bar{\lambda}\right) \subset \mathcal{D}\left(V_{K}^{1 / 2}\right)$. As any other proper extension $A_{\mathcal{V}}$ of $(A, \widetilde{A})$ that is not a restriction of $\widehat{A}$ can be characterized by a subspace $\mathcal{V}$ that without loss of generality we can assume to be contained in $(\widehat{A}-\lambda)^{-1} \operatorname{ker}\left(A^{*}-\bar{\lambda}\right) \dot{+} \operatorname{ker}\left(\widetilde{A}^{*}-\lambda\right)$, where $\mathcal{V} \not \subset(\widehat{A}-$ $\lambda)^{-1} \operatorname{ker}\left(A^{*}-\bar{\lambda}\right)$, there needs to exist at least one element in $v \in \mathcal{V}$, which is of the form $v=(\widehat{A}-\lambda)^{-1} k_{\bar{\lambda}}+\widetilde{k}_{\lambda}$, where $k_{\bar{\lambda}} \in \operatorname{ker}\left(A^{*}-\bar{\lambda}\right)$ and $\widetilde{k}_{\lambda} \in \operatorname{ker}\left(\widetilde{A}^{*}-\lambda\right)$ with $\widetilde{k}_{\lambda} \neq 0$. However, by $(4.5)$, we have that $v \notin \mathcal{D}\left(V_{K}^{1 / 2}\right)$ which implies that $A_{\mathcal{V}}$ cannot be dissipative.

Remark 4.9. A corresponding result for sectorial operators was shown in [5, Thm. 3.6.5].

Remark 4.10. In Example 4.24 below, we will discuss an operator, for which Corollary 4.8 applies.

Remark 4.11. It is not necessary that (4.5) holds in order for a dual pair to have only one proper maximally dissipative extension as we will see in Example 4.23 below.

Theorem 4.12. In addition to the assumptions of Theorem 4.7, assume that $\operatorname{dim} \mathcal{D}\left(\widetilde{A}^{*}\right) / \mathcal{D}(A)<\infty$.

Moreover, let $\mathcal{W}:=\left(\mathcal{D}\left(\widetilde{A}^{*}\right) / / \mathcal{D}(A)\right) \cap \mathcal{D}\left(V_{K}^{1 / 2}\right)$. Let the quadratic form $q$ be defined as

$$
q(w):=\operatorname{Im}\left\langle w, \widetilde{A}^{*} w\right\rangle-\left\|V_{K}^{1 / 2} w\right\|^{2},
$$

which has domain $\mathcal{W}$ and let $M$ be the selfadjoint operator associated to the unique sesquilinear form induced by $q$ by polarization. Let us decompose $\mathcal{W}=$ $\mathcal{W}_{+} \oplus \mathcal{W}_{0} \oplus \mathcal{W}_{-}$, where $\mathcal{W}_{+}$denotes the positive spectral subspace, $\mathcal{W}_{0}$ denotes $\operatorname{ker} M$ and $\mathcal{W}_{-}$denotes the negative spectral subspace of $M$. Furthermore, define

$$
M_{ \pm}:= \pm M P_{\mathcal{W}_{ \pm}}
$$

which allows us to write $M=M_{+}-M_{-}$. Note that $M_{ \pm}>0$ and that $M_{+}$and $M_{-}$are invertible on $\mathcal{W}_{+}$, resp. on $\mathcal{W}_{-}$. Let $C$ be a contraction $(\|C\| \leq 1)$ from $\mathcal{W}_{+} \oplus \mathcal{W}_{0}$ into $\mathcal{W}_{-}$. Then, there is a one-one correspondence between all pairs $(\mathfrak{M}, C)$, where $\mathfrak{M}$ is a subspace of $\mathcal{W}_{+} \oplus \mathcal{W}_{0}$ and $C$ is a contraction from $\mathcal{W}_{+}$into $\mathcal{W}_{-}$with $\mathcal{D}(C)=P_{\mathcal{W}_{+}} \mathfrak{M}$ and all proper dissipative extensions of $A$ via

$$
\begin{aligned}
\mathcal{D}\left(A_{\mathfrak{M}, C}\right) & =\mathcal{D}(A) \dot{+}\left\{w+{\sqrt{M_{-}}}^{-1} C \sqrt{M_{+}} w, w \in \mathfrak{M}\right\} \\
A_{\mathfrak{M}, C} & =\widetilde{A}^{*}\left\lceil\mathcal{D}\left(A_{\mathfrak{M}, C}\right)\right.
\end{aligned}
$$

Moreover, for an extension $\mathcal{D}\left(A_{\mathfrak{M}, C}\right)$ to be maximally dissipative, it is necessary that $\mathfrak{M}=\mathcal{W}_{+} \oplus \mathcal{W}_{0}$. 
Proof. By virtue of Theorem 4.7, we firstly need to show that

$$
q(v) \geq 0 \quad \text { for all } \quad v \in\left\{w+{\sqrt{M_{-}}}^{-1} C \sqrt{M_{+}} w, w \in \mathfrak{M}\right\}
$$

if $C$ is a contraction. By definition of $M$ and $M_{ \pm}$, we have that

$$
\begin{aligned}
q(v) & =\langle v, M v\rangle=\left\langle w+{\sqrt{M_{-}}}^{-1} C \sqrt{M_{+}} w, M\left(w+{\sqrt{M_{-}}}^{-1} C \sqrt{M_{+}} w\right)\right\rangle \\
& =\left\langle w, M_{+} w\right\rangle-\left\langle w, \sqrt{M_{+}} C^{*}{\sqrt{M_{-}}}^{-1} M_{-}{\sqrt{M_{-}}}^{-1} C \sqrt{M_{+}} w\right\rangle \\
& =\left\langle w, \sqrt{M_{+}}\left(\mathbb{1}-C^{*} C\right) \sqrt{M_{+}} w\right\rangle=\left\langle\sqrt{M_{+}} w,\left(\mathbb{1}-C^{*} C\right) \sqrt{M_{+}} w\right\rangle,
\end{aligned}
$$

which is non-negative if $C$ is a contraction on $\sqrt{M_{+}} \mathfrak{M}=P_{\mathcal{W}_{+}} \mathfrak{M}=\mathcal{D}(C)$. Let us now show that any proper dissipative extension has to be of this form. To this end, let $A^{\prime}$ be a proper dissipative extension of $(A, \widetilde{A})$ and let $\mathfrak{M}^{\prime} \subset \mathcal{W}$ be such that $\mathcal{D}\left(A^{\prime}\right) / / \mathcal{D}(A)=\mathfrak{M}^{\prime}$. Clearly, $\mathcal{W}_{-} \cap \mathfrak{M}^{\prime}=\{0\}$, since otherwise we would have that

$$
q(w)=\langle w, M w\rangle=-\left\langle w, M_{-} w\right\rangle<0
$$

for some non-zero $w \in \mathcal{W}_{-} \cap \mathfrak{M}^{\prime}$, which would violate the necessary condition as obtained from Theorem 4.7 for $A^{\prime}$ to be dissipative. This means that any $w \in \mathfrak{M}^{\prime}$ can be written as $w=w_{-}^{\perp}+w_{-}$where $w_{-}^{\perp} \in \mathcal{W}_{+} \oplus \mathcal{W}_{0}, w_{-}^{\perp} \neq 0$ and $w_{-} \in \mathcal{W}_{-}$is possibly zero. Since $\mathcal{W}_{-} \cap \mathfrak{M}=\{0\}$, it is easy to see that $w_{-}$is uniquely determined by $w_{-}^{\perp}$. Therefore, there exists a linear operator $B: P_{\mathfrak{M}^{\prime}}\left(\mathcal{W}_{+} \oplus \mathcal{W}_{0}\right) \rightarrow \mathcal{W}_{-}$such that $w=w_{-}^{\perp}+B w_{-}^{\perp}$ for any $w \in \mathfrak{M}^{\prime}$ Next observe that if for any such $w_{-}^{\perp}$ we have that $w_{-}^{\perp} \in \mathcal{W}_{0}$, it follows that $B w_{-}^{\perp}=0$. If this were not true, we would get

$$
q\left(w_{-}^{\perp}+B w_{-}^{\perp}\right)=\underbrace{\left\langle w_{-}^{\perp}, M_{+} w_{-}^{\perp}\right\rangle}_{=0}-\left\langle B w_{-}^{\perp}, M_{-} B w_{-}^{\perp}\right\rangle=-\left\langle B w_{-}^{\perp}, M_{-} B w_{-}^{\perp}\right\rangle,
$$

which again would violate the necessary condition from Theorem 4.7 for $A^{\prime}$ to be dissipative. Plugging this into the quadratic form $q$ yields:

$$
\begin{aligned}
& q\left(w_{-}^{\perp}+B w_{-}^{\perp}\right) \\
& =\left\langle w_{-}^{\perp}, M_{+} w_{-}^{\perp}\right\rangle-\left\langle B w_{-}^{\perp}, M_{-} B w_{-}^{\perp}\right\rangle=\left\langle w_{-}^{\perp},\left(M_{+}-B^{*} M_{-} B\right) w_{-}^{\perp}\right\rangle \\
& =\left\langle\sqrt{M_{+}} w_{-}^{\perp},\left(1-{\sqrt{M_{+}}}^{-1} B^{*} \sqrt{M_{-}} \sqrt{M_{-}} B \sqrt{M_{+}}-1\right) \sqrt{M_{+}} w_{-}^{\perp}\right\rangle,
\end{aligned}
$$

with the understanding that ${\sqrt{M_{+}}}^{-1}$ is defined only on $\operatorname{ran} \sqrt{M_{+}}=\operatorname{ran} M_{+}$. This is equivalent to saying that the operator $C:={\sqrt{M_{-}}}_{B}{\sqrt{M_{+}}}^{-1}$ is a contraction on $\sqrt{M_{+}} P_{\mathcal{W}_{+}} \mathfrak{M}^{\prime}=P_{\mathcal{W}_{+}} \mathfrak{M}^{\prime}$, or equivalently, $B=\sqrt{M_{-}}{ }^{-1} C \sqrt{M_{+}}$, with $C$ being a contraction from $P_{\mathcal{W}_{+}} \mathfrak{M}^{\prime}$ to $\mathcal{W}_{-}$. The condition that $\mathfrak{M}=$ $\mathcal{W}_{+} \oplus \mathcal{W}_{0}$ for $A_{\mathfrak{M}, C}$ to be maximally dissipative follows from the fact that one could always extend the operator $A_{\mathfrak{M}, C}$ to $A_{\mathcal{W}_{+} \oplus \mathcal{W}_{0}, \widehat{C}}$, where $\widehat{C}$ is an extension of $C$ which is just set equal to zero on $\left(\mathcal{W}_{+} \oplus \mathcal{W}_{0}\right) \ominus \mathfrak{M}$.

Remark 4.13. For the case that the dual pair $(A, \widetilde{A})$ has only one unique maximally dissipative proper extension $\widehat{A}$, this means that $\widehat{A}=A_{\mathcal{W}_{+}} \oplus \mathcal{W}_{0}, 0$. 
In particular, for the case that the assumptions of Corollary 4.8 are satisfied, we get that $\mathcal{W}_{-}=\{0\}$ since $\left(\mathcal{D}\left(\widetilde{A}^{*}\right) / / \mathcal{D}(A)\right) \cap \mathcal{D}\left(V_{K}^{1 / 2}\right)=\mathcal{W}_{+} \oplus \mathcal{W}_{0}$.

Remark 4.14. Let us show that for a very special situation, the spaces $\mathcal{W}_{ \pm}$ coincide with the defect spaces of a symmetric operator $S$. (As an example, take the momentum operator $i \frac{d}{d x}$ with domain $\left\{f \in H^{1}(\mathbb{R}), f(0)=0\right\}$, whose defect spaces are one-dimensional and spanned by exponential functions supported on different half-lines.) Assume that $S$ has finite-dimensional defect spaces $\mathcal{N}_{ \pm}:=\operatorname{ker}\left(S^{*} \mp i\right)$. It is a well-known fact [43] that

$$
\mathcal{D}\left(S^{*}\right)=\mathcal{D}(S) \dot{+} \mathcal{N}_{+} \dot{+} \mathcal{N}_{-},
$$

where $\mathcal{N}_{ \pm}:=\operatorname{ker}\left(S^{*} \mp i\right)$ are the defect spaces. Assume in addition the rather restrictive condition that $\mathcal{N}_{+} \perp \mathcal{N}_{-}$(orthogonal with respect to the Hilbert space inner product). Choosing the dual pair $(S, S)$, which trivially has the common core property, we find that $V_{K}=0_{\mathcal{H}}$, with $V_{K}$ being defined as in Theorem 4.7. Define

$$
q(v):=\operatorname{Im}\left\langle v, S^{*} v\right\rangle \quad \text { with } \quad v \in \mathcal{N}_{+} \oplus \mathcal{N}_{-} .
$$

A calculation shows that the operator $M$ associated to $q$ is given by $M=$ $P_{\mathcal{N}_{+}}-P_{\mathcal{N}_{-}}$, i.e. $M_{ \pm}=P_{\mathcal{N}_{ \pm}}, \mathcal{W}_{ \pm}=\mathcal{N}_{ \pm}$and $\mathcal{W}_{0}=\{0\}$. Thus, by Theorem 4.12 , all maximally dissipative extensions of such an operator $S$ are given by

$$
\mathcal{D}\left(S_{C}\right)=\mathcal{D}(S) \dot{+}\left\{n_{+}+C n_{+}, n_{+} \in \mathcal{N}_{+}\right\}, \quad S_{C}=S^{*} \Gamma_{\mathcal{D}\left(S_{C}\right)},
$$

where $C$ is any contraction into $\mathcal{N}_{-}$such that $\mathcal{D}(C)=\mathcal{N}_{+}$. Thus, for the very special case $\mathcal{N}_{+} \perp \mathcal{N}_{-}$, this readily implies the von Neumann theory of selfadjoint/maximally dissipative extensions of symmetric operators. (cf. e.g. [43, Thm. 8.12], for the selfadjoint and [6, Theorem 2.4], for the more general maximally dissipative case)

Remark 4.15. For concrete problems, it seems to be not very practical to construct $\mathcal{W}_{+}, \mathcal{W}_{0}$ and $\mathcal{W}_{-}$as well as $M_{+}$and $M_{-}$. However, this result allows us to calculate the number of independent complex parameters one can expect to describe all proper maximally dissipative extensions of a dual pair, which is given by the number of parameters that describe all contractions $C$ from $\mathcal{W}_{+}$into $\mathcal{W}_{-}$, which is equal to $\operatorname{dim} \mathcal{W}_{+} \cdot \operatorname{dim} \mathcal{W}_{-}$.

See also the operators considered in Example 4.2.3 for a discussion of the spaces $\mathcal{W}_{+}, \mathcal{W}_{-}$and $\mathcal{W}_{0}$ for a few concrete examples.

Remark 4.16. As a reference to [34], let us point out that this result means that we can characterize all proper dissipative extensions of such a dual pair using the terminology of operator balls. For any three operators $Z, R_{l}, R_{r} \in$ $\mathcal{B}(\mathcal{E})$, where $\mathcal{E}$ is an arbitrary Hilbert space, recall that the set of all operators $K \in \mathcal{B}(\mathcal{E})$ such that there exists a contraction $C$ from $\operatorname{ran} R_{r}$ to $\mathcal{D}\left(R_{l}\right)$ such that

$$
K=Z+R_{l} C R_{r}
$$

is called an operator ball $\mathfrak{B}\left(Z, R_{l}, R_{r}\right)$ with center point $Z$, left radius $R_{l}$ and right radius $R_{r}$. With the identification $\mathcal{E}=\mathcal{W}, Z=P_{\mathcal{W}_{+}}+P_{\mathcal{W}_{0}}, R_{l}=$ 
${\sqrt{M_{-}}}^{-1}$ and $R_{r}=\sqrt{M_{+}}$defined on $\mathcal{W}_{-}$, respectively on $\mathcal{W}_{+}$and the result from Theorem 4.12, we can characterize all proper dissipative extensions of a dual pair $(A, \widetilde{A})$ satisfying the assumptions of Theorem 4.12 via:

$$
A_{K}: \quad \mathcal{D}\left(A_{K}\right)=\mathcal{D}(A) \dot{+}\{K w: w \in \mathcal{W}\}, \quad A_{K}=\widetilde{A}^{*}\left\lceil\mathcal{D}\left(A_{K}\right),\right.
$$

where $K \in \mathfrak{B}\left(P_{\mathcal{W}_{+}}+P_{\mathcal{W}_{0}},{\sqrt{M_{-}}}^{-1}, \sqrt{M_{+}}\right)$.

\subsection{The non-common core case}

Let us now extend this idea to the case where the dual pair $(A, \widetilde{A})$ does not have the common core property. If we assume $\mathcal{D}(A) \cap \mathcal{D}(\widetilde{A})$ to still be dense, we can restrict $A$ and $\widetilde{A}$ to $\mathcal{D}(A) \cap \mathcal{D}(\widetilde{A})$ to obtain a dual pair of operators which has the common core property:

Corollary 4.17. Let $A$ and $\widetilde{A}$ be a dual pair of operators, where $A$ is dissipative. Moreover, let $\mathcal{D}(A) \cap \mathcal{D}(\widetilde{A})$ be dense in $\mathcal{H}$. Define the operators $A^{\prime}$ and $\widetilde{A}^{\prime}$ as follows:

$$
A^{\prime}:=\overline{A \uparrow_{\mathcal{D}(A) \cap \mathcal{D}(\widetilde{A})}} \quad \text { and } \quad \widetilde{A}^{\prime}:=\overline{\widetilde{A} \uparrow_{\mathcal{D}(A) \cap \mathcal{D}(\widetilde{A})}} .
$$

Furthermore, let $V_{0}^{\prime}$ denote the operator $\frac{1}{2 i}\left(A^{\prime}-\widetilde{A}^{\prime}\right)$ on $\mathcal{D}(A) \cap \mathcal{D}(\widetilde{A})$ and $V_{K}^{\prime}$ its corresponding Kre ̌n extension.

Now, let $\mathcal{V} \subset \mathcal{D}\left(\widetilde{A}^{\prime *}\right) / / \mathcal{D}\left(A^{\prime}\right)$ be a subspace. The operator $A_{\mathcal{V}}^{\prime}$ is a proper dissipative extension of the dual pair $A$ and $\widetilde{A}$ if and only if all of the following conditions are satisfied

- $\mathcal{V} \subset \mathcal{D}\left(V_{K}^{\prime 1 / 2}\right)$

- $\operatorname{Im}\left\langle v, \widetilde{A}^{*} v\right\rangle \geq\left\|V_{K}^{\prime}{ }^{1 / 2} v\right\|^{2}$ for all $v \in \mathcal{V}$

- $\mathcal{D}(A) \subset \mathcal{D}\left(A_{\mathcal{V}}^{\prime}\right)$

- $\mathcal{V} \subset \mathcal{D}\left(\widetilde{A}^{*}\right)$.

Proof. Since $\mathcal{D}(A) \cap \mathcal{D}(\widetilde{A})$ is dense, the operator $A \uparrow_{\mathcal{D}(A) \cap \mathcal{D}(\widetilde{A})}$ is a densely defined dissipative operator and thus closable. Moreover, since

$$
\operatorname{Im}\langle\psi, A \psi\rangle=\operatorname{Im}\langle\widetilde{A} \psi, \psi\rangle=-\operatorname{Im}\langle\psi, \widetilde{A} \psi\rangle \geq 0 \quad \text { for all } \quad \psi \in \mathcal{D}(A) \cap \mathcal{D}(\widetilde{A}),
$$

this shows that $\widetilde{A}\left\lceil_{\mathcal{D}(A) \cap \mathcal{D}(\widetilde{A})}\right.$ is a densely defined anti-dissipative operator. Thus, by construction, the operators $A^{\prime}$ and $\widetilde{A}^{\prime}$ are closed operators, which have the common core property. Moreover,

$$
A^{\prime} \subset A \subset \widetilde{A}^{*} \subset \widetilde{A}^{\prime *},
$$

from which follows that any proper dissipative extension of the dual pair $(A, \widetilde{A})$ is a proper extension of the dual pair $\left(A^{\prime}, \widetilde{A}^{\prime}\right)$ as well. The corollary now follows from the observation that its first two conditions just correspond to an application of Theorem 4.7 for the dual pair $\left(A^{\prime}, \widetilde{A}^{\prime}\right)$ (which has the common core property) to ensure that $A_{\mathcal{V}}^{\prime}$ is a dissipative extension of $A^{\prime}$. The latter two conditions ensure that $A_{\mathcal{V}}^{\prime}$ is not just a proper extension of the dual pair $\left(A^{\prime}, \widetilde{A}^{\prime}\right)$ but also of $(A, \widetilde{A})$. 
Remark 4.18. Since the dual pair $\left(A^{\prime}, \widetilde{A}^{\prime}\right)$ has the common core property and $A$ is a proper dissipative extension of this dual pair, Theorem 4.12 implies that there exists a contraction $C$ from $\mathcal{W}_{+}^{\prime}$ into $\mathcal{W}_{-}^{\prime}$ and a subspace $\mathfrak{M}^{\prime} \subset \mathcal{W}_{+}^{\prime} \oplus \mathcal{W}_{0}^{\prime}$ such that $A=A_{\mathfrak{M}^{\prime}, C}^{\prime}$, where the notation is the same as employed in (4.7). As any proper dissipative extension of the dual pair $(A, \widetilde{A})$ has to be a proper dissipative extension of $\left(A^{\prime}, \widetilde{A}^{\prime}\right)$ as well, for which Theorem 4.12 applies, this means that the problem of finding the proper dissipative extensions of $(A, \widetilde{A})$ is equivalent to determining $(\mathfrak{N}, \widehat{C})$, where $\mathfrak{M}^{\prime} \subset \mathfrak{N}$ and $\widehat{C}$ is a contractive extension of $C$ with the additional constraint that $A_{\mathfrak{N}, \widehat{C}} \subset \widetilde{A}^{*}$. For a full discussion of determining the contractive extensions of a given contraction, see $[9]$.

\subsection{Illustrating examples}

In the following, we are going to apply our results to various ODE examples, which we have chosen to illustrate our results without having to worry too much about technicalities.

4.2.1. Weakly perturbed symmetric operators. As a first application of Theorem 4.7, let us consider dual pairs of operators of the form $A=S+i V$ and $\widetilde{A}=S-i V$, where $S$ is closed and symmetric and $V$ is a positive symmetric operator, which has $S^{*}$-bound less than one. ${ }^{1}$

Theorem 4.19. Let $S$ be a closed symmetric operator and $V$ be a non-negative symmetric operator with $S^{*}$-bound less than 1 . Moreover, let $\mathfrak{d}(A, \widetilde{A})$ denote the set of proper dissipative extensions of the dual pair $(A, \widetilde{A})$. Then, the set of all proper dissipative extensions of the dual pair $S+i V$ and $S-i V$ is given by

$$
\mathfrak{d}(S+i V, S-i V)=\{\widehat{S}+i V ; \widehat{S} \in \mathfrak{d}(S, S)\} .
$$

Proof. Firstly, let us apply Theorem 4.7 to the dual pair $(S, S)$, where $S$ is closed and symmetric. In this case, the operator $(S-S) /(2 i)$ is identical to the zero operator on $\mathcal{D}(S)$, which has a unique bounded extension to the zero operator on the whole Hilbert space $\mathcal{H}$, i.e. $0_{\mathcal{H}}$. Thus, for any extension $S_{\mathcal{V}}$, where $\mathcal{V} \subset \mathcal{D}\left(S^{*}\right) / / \mathcal{D}(S)$, we trivially have $\mathcal{V} \subset \mathcal{D}\left(0_{\mathcal{H}}\right)=\mathcal{H}$. Thus, $\mathcal{V}$ needs only to satisfy the condition

$$
\operatorname{Im}\left\langle v, S^{*} v\right\rangle \geq 0 \quad \text { for all } \quad v \in \mathcal{V} .
$$

Next, let us consider the dual pair $(S+i V, S-i V)$. By the Hess-Kato Theorem [30, Corollary 1], we get that $(S-i V)^{*}=S^{*}+i V$, which we use together with Theorem 4.7. By relative boundedness, we therefore have $\mathcal{D}\left((S-i V)^{*}\right)=$ $\mathcal{D}\left(S^{*}\right)$ as well as $\mathcal{D}(S+i V)=\mathcal{D}(S)$, which means that we can choose $\mathcal{D}((S-$ $\left.i V)^{*}\right) / / \mathcal{D}(S+i V)=\mathcal{D}\left(S^{*}\right) / /(S)$. Now, observe that

$$
\operatorname{Im}\left\langle v,(S-i V)^{*} v\right\rangle=\operatorname{Im}\left\langle v,\left(S^{*}+i V\right) v\right\rangle=\operatorname{Im}\left\langle v, S^{*} v\right\rangle+\langle v, V v\rangle
$$

\footnotetext{
${ }^{1}$ Actually, we could consider dual pairs of the form $(S+D, S+\widetilde{D})$, where $(D, \widetilde{D})$ is a dual pair of dissipative/antidissipative perturbations, which are both relatively bounded with respect to $S^{*}$ with relative bound less than 1 .
} 
and that

$$
\langle v, V v\rangle=\left\|V_{K}^{1 / 2} v\right\|^{2} \quad \text { for all } \quad v \in \mathcal{D}\left(S^{*}\right)=\mathcal{D}\left(S^{*}+i V\right),
$$

which follows from relative boundedness of $V$ with respect to $S^{*}$. Hence, again we have that $\mathcal{V} \subset \mathcal{D}\left(V_{K}^{1 / 2}\right)$ is always satisfied for any $\mathcal{V} \subset \mathcal{D}((S-$ $\left.i V)^{*}\right) / / \mathcal{D}(S+i V)$. This implies that $\mathcal{V}$ only needs to satisfy

$$
\operatorname{Im}\left\langle v,(S-i V)^{*} v\right\rangle \geq\left\|V_{K}^{1 / 2} v\right\|^{2}
$$

which is equivalent to

$$
\operatorname{Im}\left\langle v, S^{*} v\right\rangle \geq 0 \text { for all } v \in \mathcal{V} .
$$

However, since this is equivalent to Condition $(4.10)$, we get that $(S+i V) \mathcal{V}$ is dissipative if and only if $S_{\mathcal{V}}$ is dissipative.

Let us start with the elementary example of a first order differential operator.

Example 4.20. Consider the closed symmetric operator on $L^{2}(0,1)$, which is given by

$$
S: \quad \mathcal{D}(S)=\left\{f \in H^{1}(0,1) ; f(0)=f(1)=0\right\}, \quad f \mapsto i f^{\prime},
$$

where $f^{\prime}$ denotes the weak derivative of $f$. Its adjoint $S^{*}$ is given by

$$
S^{*}: \quad \mathcal{D}\left(S^{*}\right)=H^{1}(0,1), \quad f \mapsto i f^{\prime} .
$$

Since for any $f \in \mathcal{D}\left(S^{*}\right)$, we have that

$$
\operatorname{Im}\left\langle f, S^{*} f\right\rangle=\frac{1}{2}\left[|f(1)|^{2}-|f(0)|^{2}\right],
$$

it follows that all dissipative extensions of $S$ are given by

$$
S_{c}: \quad \mathcal{D}\left(S_{c}\right):=\left\{f \in H^{1}(0,1) ; f(0)=c f(1)\right\}, \quad S_{c}=S^{*} \uparrow_{\mathcal{D}\left(S_{c}\right)},
$$

where $c$ is any complex number such that $|c| \leq 1$. Using Lemma 2.4, it is in fact not hard to see that these extensions are also maximal.

Moreover, let $V$ be the selfadjoint maximal multiplication operator by a nonnegative and non-zero $L^{2}$-function $V(x)$ :

$$
\begin{aligned}
V: \quad \mathcal{D}(V) & =\left\{f \in L^{2}(0,1) ; \int_{0}^{1}|V(x) f(x)|^{2} \mathrm{~d} x<\infty\right\} \\
(V f)(x) & =V(x) f(x) .
\end{aligned}
$$

For example, one could pick $V(x)=x^{-\alpha}$ with $0<\alpha<1 / 2$. Using that $H^{1}(0,1)$ compactly embeds into the bounded continuous functions $C([0,1])$ we may use that by Ehrling's Lemma there exists for any $\varepsilon>0$ a $C(\varepsilon)$ such that

$$
\|f\|_{\infty} \leq \varepsilon\left\|f^{\prime}\right\|+C(\varepsilon)\|f\|,
$$

for all $f \in H^{1}(0,1)$. This allows us to show that $V$ is $S^{*}$-bounded with $S^{*}$-bound equal to zero:

$$
\|V f\|_{2} \leq\|V\|_{2}\|f\|_{\infty} \stackrel{(4.11)}{\leq} \varepsilon\|V\|_{2}\left\|f^{\prime}\right\|_{2}+C(\varepsilon)\|V\|_{2}\|f\|_{2},
$$


where $\varepsilon\|V\|_{2}$ can be made arbitrarily small. Thus, for any non-negative $V \in$ $L^{2}(0,1)$, we may conclude that all proper dissipative extensions of the dual pair $S+i V$ and $S-i V$ are given by $S_{c}+i V$ by virtue of Theorem 4.19.

Remark 4.21. Using that $V$ is $S^{*}$-bounded with relative bound equal to zero, we have in particular that $V$ is $S_{c}$-bounded with relative bound equal to zero as well. Thus, by the Hess-Kato Theorem ([30, Corollary 1])

$$
-\left(S_{c}+i V\right)^{*}=-\left(S_{c}\right)^{*}+i V .
$$

By Proposition 2.3, we have that $-\left(S_{c}\right)^{*}$ is dissipative, which makes $-\left(S_{c}\right)^{*}+$ $i V$ dissipative. By the same proposition, we therefore may conclude that $S_{c}+i V$ is maximally dissipative.

4.2.2. Differential operators with dissipative potentials. For any $n \in \mathbb{N}$, let $p_{0}^{n}$ be the symmetric differential operator defined as follows

$$
p_{0}^{n}: \quad \mathcal{D}\left(p_{0}^{n}\right)=\mathcal{C}_{0}^{\infty}(0,1), \quad f \mapsto i^{n} f^{(n)},
$$

where $f^{(n)}$ denotes the $n^{\text {th }}$ derivative of $f$. Moreover, let $W \in L_{\text {loc }}^{2}(0,1)$ be a locally square-integrable potential function with $W \geq 0$ almost everywhere. This means that the dual pair of operators

$$
A_{0}: \quad \mathcal{D}\left(A_{0}\right)=\mathcal{C}_{0}^{\infty}(0,1), \quad\left(A_{0} f\right)(x)=i^{n} f^{(n)}(x)+i W(x) f(x)
$$

and

$$
\widetilde{A}_{0}: \quad \mathcal{D}\left(\widetilde{A}_{0}\right)=\mathcal{C}_{0}^{\infty}(0,1), \quad\left(\widetilde{A}_{0} f\right)(x)=i^{n} f^{(n)}(x)-i W(x) f(x)
$$

is well defined. Moreover, their closures $A:=\overline{A_{0}}$ and $\widetilde{A}:=\overline{\widetilde{A}_{0}}$ have the common core property by construction. In Theorem 4.7, the operator $V$ is defined as $\frac{A-\widetilde{A}}{2 i}$ on a common core $\mathcal{D} \subset(\mathcal{D}(A) \cap \mathcal{D}(\widetilde{A}))$ and we choose $\mathcal{D}=$ $\mathcal{C}_{c}^{\infty}(0,1)$. Since $V$ is already essentially selfadjoint, this implies that the Kre extension of $V$ coincides with its closure $V_{K}=\bar{V}$ and is given by the maximal multiplication operator by the function $W(x)$. Thus, $V_{K}^{1 / 2}$ is given by

$$
\begin{aligned}
V_{K}^{1 / 2}: \mathcal{D}\left(V_{K}^{1 / 2}\right) & =\left\{f \in L^{2}(0,1): \int_{0}^{1} W(x)|f(x)|^{2} \mathrm{~d} x<\infty\right\} \\
\left(V_{K}^{1 / 2} f\right)(x) & =\sqrt{W(x)} f(x) .
\end{aligned}
$$

Moreover, it can be easily shown that the domains of $A^{*}$ and $\widetilde{A}^{*}$ are given by

$$
\begin{aligned}
\widetilde{A}^{*}: \quad \mathcal{D}\left(\widetilde{A}^{*}\right) & =\left\{f \in L^{2}(0,1) ; f \in H_{\mathrm{loc}}^{n}(0,1) ; i^{n} f^{(n)}+i W f \in L^{2}\right\} \\
f & \mapsto i^{n} f^{(n)}+i W f \\
A^{*}: \quad \mathcal{D}\left(A^{*}\right) & =\left\{f \in L^{2}(0,1) ; f \in H_{\mathrm{loc}}^{n}(0,1) ; i^{n} f^{(n)}-i W f \in L^{2}\right\} \\
f & \mapsto i^{n} f^{(n)}-i W f
\end{aligned}
$$


with the understanding that $f^{(n)}$ denotes the $n^{\text {th }}$ weak derivative of $f$. By Theorem 4.7, the operator $A_{\mathcal{V}}$ (cf. Definition 4.6) is maximally dissipative, only if $\mathcal{V} \subset \mathcal{D}\left(V_{K}^{1 / 2}\right)$. Thus for any $v \in \mathcal{V}$ this implies that

$$
\int_{0}^{1}|v(x)|^{2} W(x) \mathrm{d} x<\infty
$$

and since $v \in \mathcal{D}\left(\widetilde{A}^{*}\right) \subset L^{2}(0,1)$, which implies that $i^{n} v^{(n)}+i W v \in L^{2}(0,1)$, it follows that

$$
\overline{v(x)} i^{n} v^{(n)}(x)+i|v(x)|^{2} W(x) \in L^{1}(0,1)
$$

from which - together with (4.14) and an application of the reverse triangle inequality - it follows that

$$
\int_{0}^{1}\left|\overline{v(x)} i^{n} v^{(n)}(x)\right| \mathrm{d} x<\infty,
$$

i.e. $\bar{v} v^{(n)} \in L^{1}(0,1)$. Hence, given that $v \in \mathcal{D}\left(V_{K}^{1 / 2}\right)$ the necessary and sufficient condition for $A_{\mathcal{V}}$ to be dissipative

$$
\operatorname{Im}\left\langle v, \widetilde{A}^{*} v\right\rangle \geq\left\|W^{1 / 2} v\right\|^{2} \text { for all } v \in \mathcal{V}
$$

simplifies to

$$
\operatorname{Im}\left\langle v, i^{n} v^{(n)}\right\rangle \geq 0 \quad \text { for all } \quad v \in \mathcal{V}
$$

4.2.3. First order differential operators with singular potentials. Let us apply the result of the previous subsection to the simplest case $n=1$. For any $\varepsilon>0$, any $x_{0} \in(0,1)$ and any $v \in H_{\text {loc }}^{1}(0,1)$ we have that

$$
|v(\varepsilon)|^{2}=\left|v\left(x_{0}\right)\right|^{2}-2 \operatorname{Im} \int_{\varepsilon}^{x_{0}} \overline{v(x)} i v^{\prime}(x) \mathrm{d} x
$$

and since $\bar{v} v^{\prime} \in L^{1}$, we have by an explicit calculation

$$
\begin{aligned}
\lim _{\varepsilon \downarrow 0}|v(\varepsilon)|^{2} & =\lim _{\varepsilon \downarrow 0}\left(\left|v\left(x_{0}\right)\right|^{2}-2 \operatorname{Im} \int_{\varepsilon}^{x_{0}} \overline{v(x)} i v^{\prime}(x) \mathrm{d} x\right) \\
& =\left|v\left(x_{0}\right)\right|^{2}-2 \operatorname{Im} \int_{0}^{x_{0}} \overline{v(x)} i v^{\prime}(x) \mathrm{d} x .
\end{aligned}
$$

The same reasoning can be applied to show the existence of $\lim _{\varepsilon \downarrow 0}|v(1-\varepsilon)|^{2}$, which shows that $|v|^{2}$ is continuous up to the boundary of the interval. Defining, at least formally,

$$
|v(0)|^{2}:=\lim _{\varepsilon \downarrow 0}|v(\varepsilon)|^{2} \quad \text { and } \quad|v(1)|^{2}:=\lim _{\varepsilon \downarrow 0}|v(1-\varepsilon)|^{2}
$$

we get that

$$
\operatorname{Im}\left\langle v, i v^{\prime}\right\rangle=\frac{1}{2}\left(|v(1)|^{2}-|v(0)|^{2}\right) \quad \text { for all } \quad v \in H_{\mathrm{loc}}^{1}(0,1): \bar{v} v^{\prime} \in L^{1} .
$$

Let us now consider a few different potentials: 
Example 4.22. Let $1 / 2 \leq \alpha<1$ and let the potential function be given by $W(x)=\frac{1-\alpha}{x^{\alpha}}$, where the numerator $(1-\alpha)$ is chosen for convenience (the case $0<\alpha<1 / 2$ has been covered in Example 4.20). By an explicit calculation, it can be shown that

$$
\mathcal{D}\left(\widetilde{A}^{*}\right)=\mathcal{D}(A) \dot{+} \operatorname{span}\left\{\exp \left(-x^{1-\alpha}\right), \exp \left(-x^{1-\alpha}\right) \int_{0}^{x} \exp \left(2 t^{1-\alpha}\right) \mathrm{d} t\right\}
$$

and it is easy to see that

$$
\begin{aligned}
\mathcal{D}\left(\widetilde{A}^{*}\right) / / \mathcal{D}(A) & =\operatorname{span}\left\{\exp \left(-x^{1-\alpha}\right), \exp \left(-x^{1-\alpha}\right) \int_{0}^{x} \exp \left(2 t^{1-\alpha}\right) \mathrm{d} t\right\} \\
& \subset \mathcal{D}\left(V_{K}^{1 / 2}\right)=\mathcal{D}\left(x^{-\frac{\alpha}{2}}\right),
\end{aligned}
$$

where the last inclusion is guaranteed by the choice $\alpha<1$. A standard linear transformation shows that it is possible to define two vectors $\phi, \psi \in$ $\mathcal{D}\left(\widetilde{A}^{*}\right) / / \mathcal{D}(A)$ such that

$$
\mathcal{D}\left(\widetilde{A}^{*}\right)=\mathcal{D}(A) \dot{+} \operatorname{span}\{\phi, \psi\}
$$

and $\phi, \psi$ satisfy the boundary conditions

$$
\psi(0)=1, \psi(1)=0, \phi(0)=0, \phi(1)=1 .
$$

Thus, if we choose two complex numbers $\left(c_{1}, c_{2}\right) \in \mathbb{C}^{2} \backslash\{(0,0)\}$ in order to parametrize all one-dimensional proper extensions of $(A, \widetilde{A})$ as

$$
A_{c_{1}, c_{2}}: \quad \mathcal{D}\left(A_{c_{1}, c_{2}}\right)=\mathcal{D}(A) \dot{+} \operatorname{span}\left\{c_{1} \phi+c_{2} \psi\right\}, \quad A_{c_{1}, c_{2}}=\widetilde{A}^{*}\left\lceil\mathcal{D}\left(A_{c_{1}, c_{2}}\right)\right.
$$

and plug $v_{c_{1}, c_{2}}:=c_{1} \phi+c_{2} \psi$ into (4.17), we get the condition that

$$
\operatorname{Im}\left\langle v_{c_{1}, c_{2}}, i v_{c_{1}, c_{2}}^{\prime}\right\rangle=\frac{1}{2}\left(\left|c_{1}\right|^{2}-\left|c_{2}\right|^{2}\right) \geq 0,
$$

i.e. $\left|c_{1}\right| \geq\left|c_{2}\right|$. Thus, we can parametrize all maximally dissipative proper extensions using only one complex parameter $c=c_{2} / c_{1}$ with $|c| \leq 1$ and get $\left\{A_{c}:|c| \leq 1\right\}$, where

$$
A_{c}: \quad \mathcal{D}\left(A_{c}\right)=\mathcal{D}(A) \dot{+} \operatorname{span}\{\phi+c \psi\}, \quad A_{c}=\widetilde{A}^{*}\left\lceil\mathcal{D}\left(A_{c}\right)\right.
$$

as a complete description of the set of all proper maximally dissipative extensions.

Let us now consider examples, where the singularity of the potential is of "same strength" as the differential operator $(\alpha=1)$.

Example 4.23. Let $0<\gamma<1 / 2$ and consider the potential

$$
W(x)=\frac{\gamma}{1-x} .
$$

Note that this is equivalent to considering the operator $-i \frac{\mathrm{d}}{\mathrm{d} y}+i \frac{\gamma}{y}$ after the coordinate change $(1-x) \mapsto y$, which leads to a change of sign in front of the differential part of the operator, changing the situation significantly 
compared to Example 4.24.

In this case, a calculation shows that for our range of $\gamma$, we have

$$
\mathcal{D}\left(\widetilde{A}^{*}\right)=\mathcal{D}(A) \dot{+} \operatorname{span}\left\{(1-x)^{\gamma},(1-x)^{1-\gamma}\right\} .
$$

Since $0<\gamma<1 / 2$, it is true that

$$
\operatorname{span}\left\{(1-x)^{\gamma},(1-x)^{1-\gamma}\right\} \subset \mathcal{D}\left(V_{K}^{1 / 2}\right)=\mathcal{D}\left(\frac{1}{\sqrt{1-x}}\right)
$$

and $\operatorname{dim} \operatorname{ker} A^{*}=1$, all proper dissipative extensions of $A$ will be at most one-dimensional extensions, i.e. of the form

$$
\mathcal{D}\left(A_{c_{1}, c_{2}}\right):=\mathcal{D}(A) \dot{+} \operatorname{span}\left\{c_{1}(1-x)^{\gamma}+c_{2}(1-x)^{1-\gamma}\right\},
$$

where $\left(c_{1}, c_{2}\right) \in \mathbb{C}^{2} \backslash\{(0,0)\}$. Plugging $v_{c_{1}, c_{2}}:=c_{1}(1-x)^{\gamma}+c_{2}(1-x)^{1-\gamma}$ into Equation (4.17), we get the condition

$$
\operatorname{Im}\left\langle v_{c_{1}, c_{2}}, i v_{c_{1}, c_{2}}^{\prime}\right\rangle=-\frac{\left|c_{1}+c_{2}\right|^{2}}{2} \geq 0
$$

which is satisfied if and only if $c_{1}=-c_{2}$. Thus, there exists a unique proper maximally dissipative extension of the dual pair $(A, \widetilde{A})$, which is given by

$$
A^{\prime}: \quad \mathcal{D}\left(A^{\prime}\right)=\mathcal{D}(A) \dot{+} \operatorname{span}\left\{(1-x)^{\gamma}-(1-x)^{1-\gamma}\right\}, \quad A^{\prime}=\widetilde{A}^{*}\left\lceil\mathcal{D}\left(A^{\prime}\right) .\right.
$$

This is an example of a dual pair $(A, \widetilde{A})$ with a unique proper maximally dissipative extension, which does not satisfy the assumptions of Corollary 4.8 .

Next, let us compute the spaces $\mathcal{W}_{+}, \mathcal{W}_{0}$ and $\mathcal{W}_{-}$as defined in Theorem 4.12. Since the form $q$ as defined in Equation (4.6) is given by

$$
q(v)=\operatorname{Im}\left\langle v, i v^{\prime}\right\rangle=\frac{1}{2}\left(|v(1)|^{2}-|v(0)|^{2}\right)
$$

and is non-positive for $v \in \operatorname{span}\left\{(1-x)^{\gamma},(1-x)^{1-\gamma}\right\}$ by virtue of Equation (4.18), we have found the maximizer of $\langle v, M v\rangle$ which corresponds to the eigenvalue zero:

$$
\mathcal{W}_{0}=\operatorname{ker} M=\operatorname{span}\left\{(1-x)^{\gamma}-(1-x)^{1-\gamma}\right\}
$$

and - using the Gram-Schmidt procedure - we compute

$$
\mathcal{W}_{-}=\operatorname{span}\{\underbrace{\left(4 \gamma^{2}-8 \gamma-5\right)(1-x)^{\gamma}-\left(4 \gamma^{2}-8 \gamma+3\right)(1-x)^{1-\gamma}}_{=: w_{-}}\}
$$

with eigenvalue

$$
\lambda_{-}=\frac{\left\langle w_{-}, M w_{-}\right\rangle}{\left\langle w_{-}, w_{-}\right\rangle}=\frac{\frac{1}{2}\left(\left|w_{-}(1)\right|^{2}-\left|w_{-}(0)\right|^{2}\right)}{\int_{0}^{1}\left|w_{-}(x)\right|^{2} \mathrm{~d} x}=-\frac{2}{-4 \gamma^{2}+4 \gamma+7} .
$$

Example 4.24. Let $0<\gamma<1 / 2$ and consider the potential

$$
W(x)=\frac{\gamma}{x} .
$$


In this case, a calculation shows that $\mathcal{D}\left(\widetilde{A}^{*}\right)=\mathcal{D}(A) \dot{+} \operatorname{span}\left\{x^{-\gamma}, x^{1+\gamma}\right\}$. This is an example, for which Corollary 4.8 applies, since $\operatorname{ker} \widetilde{A}^{*}=\operatorname{span}\left\{x^{-\gamma}\right\}$ has trivial intersection with $\mathcal{D}\left(V_{K}^{1 / 2}\right)=\left\{f \in L^{2}(0,1), \int_{0}^{1}|f(x)|^{2} x^{-1} \mathrm{~d} x<\right.$ $\infty\}$. Hence, the only possible candidate for a proper maximally dissipative extension for the dual pair $(A, \widetilde{A})$ is the operator $\widehat{A}$, which is given by

$$
\widehat{A}: \quad \mathcal{D}(\widehat{A})=\mathcal{D}(A) \dot{+} \operatorname{span}\left\{x^{1+\gamma}\right\}, \quad \widehat{A}=\widetilde{A}^{*} \uparrow_{\mathcal{D}(\widehat{A})} .
$$

By Proposition 2.8 , it is already clear that $\widehat{A}$ has to be a proper maximally dissipative extension. This can also be verified explicitely by by plugging $v(x):=x^{1+\gamma}$ into Condition (4.17).

In this concrete case, we have that $\mathcal{W}_{0}=\mathcal{W}_{-}=\{0\}$ and $\mathcal{W}_{+}=\operatorname{span}\left\{x^{1+\gamma}\right\}$. A short calculation shows that the corresponding eigenvalue of $M$ is given by

$$
\lambda_{+}=\frac{\left\langle x^{1+\gamma}, M x^{1+\gamma}\right\rangle}{\left\langle x^{1+\gamma}, x^{1+\gamma}\right\rangle}=\frac{3}{2}+\lambda .
$$

4.2.4. A second order example. Let us now apply our results to an example, where the operator $V$ as defined in the statement of Theorem 4.7 is not essentially selfadjoint. To this end, consider the dual pair of operators given by

$$
\begin{array}{lll}
A_{0}: & \mathcal{D}\left(A_{0}\right)=\mathcal{C}_{c}^{\infty}(0,1), \quad\left(A_{0} f\right)(x)=-i f^{\prime \prime}(x)-\gamma \frac{f(x)}{x^{2}}, \\
\widetilde{A}_{0}: & \mathcal{D}\left(\widetilde{A}_{0}\right)=\mathcal{C}_{c}^{\infty}(0,1), \quad\left(\widetilde{A}_{0} f\right)(x)=i f^{\prime \prime}(x)-\gamma \frac{f(x)}{x^{2}} .
\end{array}
$$

Since we have

$$
\operatorname{Im}\left\langle f, A_{0} f\right\rangle=\operatorname{Im} \int_{0}^{1} \overline{f(x)}\left(-i f^{\prime \prime}(x)-\gamma \frac{f(x)}{x^{2}}\right) \mathrm{d} x=\int_{0}^{1}\left|f^{\prime}(x)\right|^{2} \mathrm{~d} x
$$

for all $f \in \mathcal{C}_{c}^{\infty}(0,1)$, we can estimate $\operatorname{Im}\left\langle f, A_{0} f\right\rangle$ from below by the lowest eigenvalue of the Dirichlet-Laplacian on the unit interval, which is $\pi^{2}$, i.e.

$$
\operatorname{Im}\left\langle f, A_{0} f\right\rangle \geq \pi^{2}\|f\|^{2} \quad \text { for all } \quad f \in \mathcal{D}\left(A_{0}\right) .
$$

Now, define $A:=\overline{A_{0}}$ and $\widetilde{A}:=\overline{\widetilde{A}_{0}}$, which means that the dual pair $(A, \widetilde{A})$ has the common core property by construction. Also, (4.19) implies in particular that $0 \in \widehat{\rho}(A)$. By a simple calculation, it can be shown that the operator $\widetilde{A}^{*}$ is given by:

$$
\begin{aligned}
\widetilde{A}^{*}: \quad \mathcal{D}\left(\widetilde{A}^{*}\right) & =\left\{f \in H_{l o c}^{2}(0,1): \int_{0}^{1}\left|-i f^{\prime \prime}(x)-\gamma \frac{f(x)}{x^{2}}\right|^{2} \mathrm{~d} x<\infty\right\} \\
\left(\widetilde{A}^{*} f\right)(x) & =-i f^{\prime \prime}(x)-\gamma \frac{f(x)}{x^{2}} .
\end{aligned}
$$

A calculation, using Formula (2.1) for $\lambda=0$, yields

$$
\mathcal{D}\left(\widetilde{A}^{*}\right)=\mathcal{D}(A) \dot{+} \operatorname{span}\left\{x^{\omega}, x^{\bar{\omega}+2}\right\},
$$


where $\omega:=(1+\sqrt{1+4 i \gamma}) / 2$. Here we have assumed that $\gamma \geq \sqrt{3}$. This choice for $\gamma$ ensures that $\operatorname{dim} \operatorname{ker} \widetilde{A}^{*}=\operatorname{dim} \operatorname{ker} A^{*}=1$, which will make our calculations simpler. Also, observe that $\widetilde{A}^{*}=J A^{*} J$, where the conjugation operator $J$ is defined as $(J f)(x):=\overline{f(x)}$. From this it immediately follows that $\mathcal{D}\left(A^{*}\right)=J \mathcal{D}\left(\widetilde{A}^{*}\right)=\left\{f: \bar{f} \in \mathcal{D}\left(\widetilde{A}^{*}\right)\right\}$. Now, let us apply the result of Theorem 4.7 in order to construct maximally dissipative extensions of the dual pair $(A, \widetilde{A})$. Let $\mathcal{D}=\mathcal{C}_{c}^{\infty}(0,1)$, which is a common core for $A$ and $\widetilde{A}$ and define $V:=\frac{1}{2 i}(A-\widetilde{A})\lceil\mathcal{D}$, which is given by

$$
V: \quad \mathcal{D}(V)=\mathcal{C}_{c}^{\infty}(0,1), \quad f \mapsto-f^{\prime \prime} .
$$

As the norm induced by $\|\cdot\|_{V}:=\|\cdot\|+\langle\cdot, V \cdot\rangle$ is the $H^{1}$-norm, $\operatorname{closing} \mathcal{D}(V)=$ $\mathcal{C}_{c}^{\infty}(0,1)$ with respect to $\|\cdot\|_{V}$ yields that $\mathcal{D}\left(V_{F}^{1 / 2}\right)=H_{0}^{1}(0,1)$. Moreover, since ker $V^{*}=\operatorname{span}\{1, x\}$ and since by $(4.1)$, we have $\mathcal{D}\left(V_{K}^{1 / 2}\right)=\mathcal{D}\left(V_{F}^{1 / 2}\right) \dot{+} \operatorname{ker} V^{*}$ it is clear that $\mathcal{D}\left(V_{K}^{1 / 2}\right)=H^{1}(0,1)$ and moreover that

$$
\left\|V_{K}^{1 / 2} f\right\|^{2}=\left\|V_{F}^{1 / 2}[f(x)-f(0)-x(f(1)-f(0))]\right\|^{2}=\left\|f^{\prime}\right\|^{2}-|f(1)-f(0)|^{2},
$$

where the first equality follows from the decomposition (4.1) and the second from an explicit calculation. Using this, we can show that the form $q(v):=$ $\operatorname{Im}\left\langle v, \widetilde{A}^{*} v\right\rangle-\left\|V_{K}^{1 / 2} v\right\|^{2}$ defined on $\mathcal{D}\left(\widetilde{A}^{*}\right) / / \mathcal{D}(A)=\operatorname{span}\left\{x^{\omega}, x^{\bar{\omega}+2}\right\}$ is given by

$$
q(v)=-\operatorname{Re}\left(\overline{v(1)} v^{\prime}(1)\right)+|v(1)|^{2} .
$$

By Lemma 2.4, any maximally dissipative proper extension of $(A, \widetilde{A})$ can be parametrized by a one-dimensional subspace of $\operatorname{span}\left\{x^{\omega}, x^{\bar{\omega}+2}\right\}$. A convenient basis for this is given by the two functions

$$
\psi(x):=\frac{\left(2+\overline{\omega_{+}}\right) x^{\omega_{+}}-\omega_{+} x^{\overline{\omega_{+}}+2}}{2+\overline{\omega_{+}}-\omega_{+}} \quad \text { and } \quad \phi(x):=\frac{-x^{\omega_{+}}+x^{\overline{\omega_{+}}+2}}{2+\overline{\omega_{+}}-\omega_{+}},
$$

which satisfy the boundary conditions $\psi(1)=1, \psi^{\prime}(1)=0, \phi(1)=0$ and $\phi^{\prime}(1)=1$.

Now define $\xi_{\rho}:=\rho \psi+\phi$, where $\rho \in \mathbb{C}$ has to be determined such that $q\left(\xi_{\rho}\right) \geq 0$. A short explicit calculation shows that this is the case if and only if

$$
\left|\rho-\frac{1}{2}\right| \geq \frac{1}{2}
$$

i.e. if and only if $\rho$ lies in the exterior of the open circle with radius and center point $\frac{1}{2}$. Since $q(\psi)=1>0$, we have that $\xi_{\infty}:=\psi$ describes a maximally dissipative extension as well. Thus the set of all proper maximally dissipative extensions of $(A, \widetilde{A})$ is given by

$$
A_{\rho}: \quad \mathcal{D}\left(A_{\rho}\right)=\mathcal{D}(A) \dot{+} \operatorname{span}\left\{\xi_{\rho}\right\}, \quad A_{\rho}=\widetilde{A}^{*}\left\lceil\mathcal{D}\left(A_{\rho}\right),\right.
$$

where

$$
\rho \in\left\{z \in \mathbb{C},\left|z-\frac{1}{2}\right| \geq \frac{1}{2}\right\} \cup\{\infty\}
$$




\section{Stability of the numerical range}

Let us now prove a useful result that allows us to estimate the lower bound of the imaginary part of the numerical range of the extensions of a dual pair $(A, \widetilde{A})$ :

Lemma 5.1. Let the dual pair $(A, \widetilde{A})$ satisfy the assumptions of Theorem 4.7 and let $\mathcal{V}$ be a subspace of $\mathcal{D}\left(\widetilde{A}^{*}\right) / / \mathcal{D}(A)$ such that $\mathcal{D}\left(A_{\mathcal{V}}\right)$ is a proper dissipative extension of the dual pair $(A, \widetilde{A})$. Moreover, for $v \in \mathcal{V}$, let $q(v):=$ $\operatorname{Im}\left\langle v, \widetilde{A}^{*} v\right\rangle-\left\|V_{K}^{1 / 2} v\right\|^{2}$. Then, it is true that

$$
\operatorname{Im}\left\langle(f+v), A_{\mathcal{V}}(f+v)\right\rangle=\left\|V_{K}^{1 / 2}(f+v)\right\|^{2}+q(v) \geq\left\|V_{K}^{1 / 2}(f+v)\right\|^{2}
$$

for all $f \in \mathcal{D}(A), v \in \mathcal{V}$.

Proof. Let $f \in \mathcal{D}$ and $v \in \mathcal{V}$. As in the proof of Theorem 4.7, we use Lemma 4.1 , from which we know that it is sufficient to check the assertion for such $f$ and $v$. From an explicit calculation, we get

$$
\begin{aligned}
\operatorname{Im}\langle(f & +v), A \mathcal{V}(f+v)\rangle=\operatorname{Im}\left\langle(f+v), \widetilde{A}^{*}(f+v)\right\rangle \\
& =\operatorname{Im}\langle f, A f\rangle+\operatorname{Im}\left\langle v, \widetilde{A}^{*} v\right\rangle+\operatorname{Im}\left(\left\langle f, \widetilde{A}^{*} v\right\rangle+\left\langle v, \widetilde{A}^{*} f\right\rangle\right) \\
& =\operatorname{Im}\langle f, A f\rangle+q(v)+\left\|V_{K}^{1 / 2} v\right\|^{2}+\operatorname{Im}\left(\left\langle f, \widetilde{A}^{*} v\right\rangle+\left\langle v, \widetilde{A}^{*} f\right\rangle\right) .
\end{aligned}
$$

Now, we can use that $\operatorname{Im}\langle f, A f\rangle=\langle f, V f\rangle$, which implies in particular that $f \in \mathcal{D} \subset \mathcal{D}\left(V_{K}\right) \subset \mathcal{D}\left(V_{K}^{1 / 2}\right)$ since $V_{K}$ is a selfadjoint extension of $V$. Thus, we have that

$$
\operatorname{Im}\langle f, A f\rangle=\langle f, V f\rangle=\left\|V_{K}^{1 / 2} f\right\|^{2}
$$

and another calculation - similar as in the proof of Theorem 4.7 - shows that

$$
\operatorname{Im}\left(\left\langle f, \widetilde{A}^{*} v\right\rangle+\left\langle v, \widetilde{A}^{*} f\right\rangle\right)=2 \operatorname{Re}\left\langle V_{K}^{1 / 2} f, V_{K}^{1 / 2} v\right\rangle .
$$

Plugging these two identities back into (5.1) yields

$$
\begin{aligned}
\operatorname{Im}\left\langle(f+v), \widetilde{A}^{*}(f+v)\right\rangle & =\left\|V_{K}^{1 / 2} f\right\|^{2}+2 \operatorname{Re}\left\langle V_{K}^{1 / 2} f, V_{K}^{1 / 2} v\right\rangle+\left\|V_{K}^{1 / 2} v\right\|^{2}+q(v) \\
& =\left\|V_{K}^{1 / 2}(f+v)\right\|^{2}+q(v) .
\end{aligned}
$$

Since by Theorem 4.7 we have that $q(v) \geq 0$ for all $v \in \mathcal{V}$ it trivially follows that

$$
\operatorname{Im}\left\langle f+v, A_{\mathcal{V}}(f+v)\right\rangle \geq\left\|V_{K}^{1 / 2}(f+v)\right\|^{2}
$$

for all $f \in \mathcal{D}(A)$ and $v \in \mathcal{V}$.

Example 5.2. As a first example, consider the dual pair $(A, \widetilde{A})$ from Subsection 4.2.4, with the maximally dissipative extensions $A_{\rho}$ as described in (4.23) and (4.24). Again, it suffices to find a lower bound of $\operatorname{Im}\left\langle f+v, \widetilde{A}^{*}(f+v)\right\rangle$ for all $f \in \mathcal{C}_{c}^{\infty}(0,1)$ and all $v \in \operatorname{span}\left\{\xi_{\rho}\right\}$, where $\xi_{\rho}$ was defined in Subsection 4.2.4. Observe that

$$
\operatorname{Im}\left\langle f+v, A_{\rho}(f+v)\right\rangle=\left\|f^{\prime}+v^{\prime}\right\|^{2}-\frac{\operatorname{Re}(\rho)}{|\rho|^{2}}|v(1)|^{2}=: \mathfrak{a}(f+v)
$$


and $\mathcal{C}_{c}^{\infty}(0,1) \dot{+} \operatorname{span}\left\{\xi_{\rho}\right\} \subset \mathfrak{C}$, where $\mathfrak{C}:=\left\{f \in H^{1}(0,1): f(0)=0\right\}$. For the special cases $\rho=0$ and $\rho=\infty$, we have

$$
\operatorname{Im}\left\langle f+v, A_{\rho}(f+v)\right\rangle=\left\|f^{\prime}+v^{\prime}\right\|^{2}=: \mathfrak{a}(f+v) .
$$

Now, since $\mathfrak{C}$ equipped with the norm induced by $\mathfrak{a}$ is a Hilbert space, this implies that $\operatorname{Im}\left\langle f+v, A_{\mathcal{V}}(f+v)\right\rangle \geq \lambda_{\rho}\|f+v\|^{2}$, where $\lambda_{\rho}$ is the lowest eigenvalue of the selfadjoint operator $S_{\rho}$ associated to $(\mathfrak{a}, \mathfrak{C})$. This operator is given by

$$
\begin{aligned}
S_{\rho}: \quad \mathcal{D}\left(S_{\rho}\right) & =\left\{f \in H^{2}(0,1): f(0)=0 \text { and } f^{\prime}(1)=\frac{\operatorname{Re}(\rho)}{|\rho|^{2}} f(1)\right\} \\
f & \mapsto-f^{\prime \prime},
\end{aligned}
$$

with the understanding that the case $\rho=0$ corresponds to a Dirichlet boundary condition at one. As it is not difficult to solve the eigenvalue equation $S_{\rho} f=\lambda_{\rho} f$, where $\lambda_{\rho}$ is the smallest eigenvalue of $S_{\rho}$, one finds that $\lambda_{\rho}$ is given by $\lambda_{\rho}=z^{2}$, where $z$ is the smallest positive solution of the transcendental equation

$$
\frac{\tan z}{z}=\frac{|\rho|^{2}}{\operatorname{Re}(\rho)},
$$

where $\rho \in\{z \in \mathbb{C}: z \neq 0, \operatorname{Re}(z)=0\}$ corresponds to the singularity of $\frac{\tan z}{z}$ at $z=\frac{\pi}{2}$.

For $\operatorname{Re}(\rho)<0$, this means in particular that $\operatorname{Im}\left\langle f+v, A_{\rho}(f+v)\right\rangle \geq \frac{\pi^{2}}{4}\|f+v\|^{2}$ as can easily be seen from the fact that $(\tan z) / z$ is positive in $[0, \pi / 2)$ and non-positive in $(\pi / 2, \pi]$.

Remark 5.3. In this example, the estimate on the lower bound of the imaginary parts is also sharp. This follows from the fact that closing $\mathcal{C}_{0}^{\infty}(0,1) \dot{+} \operatorname{span}\left\{\xi_{\rho}\right\}$ with respect to the norm induced by a yields $\mathfrak{C}$ for $\rho \neq 0$ and closing $\mathcal{C}_{0}^{\infty}(0,1) \dot{+} \operatorname{span}\left\{\xi_{0}\right\}$ with respect to the $H^{1}$-norm yields $H_{0}^{1}(0,1)$.

Theorem 5.4. Let the dual pair $(A, \widetilde{A})$ satisfy the same conditions as in Theorem 4.7. If in addition we have that $\mathcal{V} \subset \mathcal{D}\left(V_{F}^{1 / 2}\right)$, we get that the imaginary part of the numerical range stays stable, i.e.

$$
\inf _{z \in \mathcal{N}_{A}} \operatorname{Im} z=\inf _{z \in \mathcal{N}_{A_{\mathcal{V}}}} \operatorname{Im} z
$$

where $\mathcal{N}_{C}$ denotes the numerical range of an operator $C$ and $A_{\mathcal{V}}$ is the extension of $A$ as described in Definition 4.6. This is true in particular for any dissipative extension of a dual pair operator $(A, \widetilde{A})$, where the associated operator $V$ is essentially selfadjoint.

Proof. For $f \in \mathcal{D}(A) \cap \mathcal{D}(\widetilde{A})$, we have that $f \in \mathcal{D}(V) \subset \mathcal{D}\left(V_{F}^{1 / 2}\right)$. Now, since by assumption $\mathcal{V} \subset \mathcal{D}\left(V_{F}^{1 / 2}\right)$, we get by virtue of Lemma 5.1 that

$$
\operatorname{Im}\left\langle(f+v), \widetilde{A}^{*}(f+v)\right\rangle \geq\left\|V_{K}^{1 / 2}(f+v)\right\|^{2}=\left\|V_{F}^{1 / 2}(f+v)\right\|^{2},
$$


for all $f \in \mathcal{D}(A) \cap \mathcal{D}(\widetilde{A})$ and for all $v \in \mathcal{V}$. Using that for all $f \in \mathcal{D}(A) \cap \mathcal{D}(\widetilde{A})$ we have that

$$
\operatorname{Im}\langle f, A f\rangle=\langle f, V f\rangle
$$

which implies that

$$
\inf _{z \in \mathcal{N}_{A}} \operatorname{Im} z=\inf _{x \in \mathcal{N}_{V}} x=\inf _{x \in \mathcal{N}_{V_{F}}} x,
$$

where the last equality follows from the fact that the numerical range of the Friedrichs extension of a semibounded operator stays stable. Using Inequality (5.3), we therefore get

$$
\inf _{z \in \mathcal{N}_{A \mathcal{V}}} \operatorname{Im} z \geq \inf _{x \in \mathcal{N}_{V_{F}}} x=\inf _{z \in \mathcal{N}_{A}} \operatorname{Im} z
$$

which together with the trivial estimate for taking the infimum over a larger set

$$
\inf _{z \in \mathcal{N}_{A \mathcal{V}}} \operatorname{Im} z \leq \inf _{z \in \mathcal{N}_{A}} \operatorname{Im} z
$$

yields the theorem.

Example 5.5. As an example, consider the operators $\left(A_{0}, \widetilde{A}_{0}\right)$ as described in Section $4.2 .2,(4.12)$ and $(4.13)$. Since the operator $V=\frac{1}{2 i}\left(A_{0}-\widetilde{A}_{0}\right)$ is given by

$$
V: \quad \mathcal{D}(V)=\mathcal{C}_{c}^{\infty}(0,1), \quad(V f)(x)=W(x) f(x),
$$

which is essentially selfadjoint, we get that $V_{K}^{1 / 2}=V_{F}^{1 / 2}=\bar{V}^{1 / 2}$ is the maximal multiplication operator by $\sqrt{W(x)}$. Hence by virtue of Theorem 5.4, we get that for any proper maximally dissipative extension $A_{\mathcal{V}}$, we have

$$
\operatorname{Im}\left\langle f+v, A_{\mathcal{V}}(f+v)\right\rangle \geq w\|f+v\|^{2},
$$

where $w:=\operatorname{essinf}_{x \in(0,1)} W(x)=\inf _{f \in \mathcal{D}(A):\|f\|=1} \operatorname{Im}\langle f, A f\rangle$.

\section{References}

[1] A. Alonso, B. Simon: The Birman-Krel̆n-Vishik theory of selfadjoint extensions of semibounded operators, J. Operator Theory 4 (1980), 251-270.

[2] A. Alonso, B. Simon: Addenda to "The Birman-Krĕn-Vishik theory of selfadjoint extensions of semibounded operators", J. Operator Theory 6 (1981), 407.

[3] T. Ando, K. Nishio: Positive selfadjoint extensions of positive symmetric operators, Tohóku Math. J., 22 (1970), 65-75.

[4] Yu. Arlinskiǔ: On Proper Accretive Extension of Positive Linear Relations, Ukrainian Mathematical Journal, 47 (1995), no. 6, 723-730.

[5] Yu. Arlinskiǔ: Boundary triplets and maximal accretive extensions of sectorial operators, Operator Methods for Boundary Value Problems. Ed. Seppo Hassi, Hendrik S. V. de Snoo, and Franciszek Hugon Szafraniec. 1st ed. Cambridge: Cambridge University Press, (2012), 35-72. 
[6] Yu. Arlinskiŭ, Yu. Kovalev and E. Tsekanovskiǔ: Accretive and Sectorial Extensions of Nonnegative Symmetric Operators, Complex Anal. Oper. Theory, 6 (2012), 677-718.

[7] Yu. Arlinskiı̌ and E. Tsekanovskiǐ: The von Neumann problem for nonnegative symmetric operators, Integral Equations Operator Theory, 51 (2005), 319-356.

[8] Yu. Arlinskiı̌ and E. Tsekanovskiǔ: M. Kreŭn's Research on Semi-Bounded Operators, its Contemporary Developments and Applications, Oper. Theory Adv. and Appl., 190 (2009), 65-112.

[9] Gr. Arsene and A. Gheondea: Completing matrix contractions, J. Operator Theory 7 (1982), 179-189.

[10] M.S.Birman: On the selfadjoint extensions of positive definite operators, Mat. Sbornik 38 (1956), 431-450 (Russian).

[11] B.M. Brown and W.D. Evans: Selfadjoint and $m$-sectorial extensions of SturmLiouville operators, submitted.

[12] B. M. Brown, G. Grubb and I. Wood: $M$-functions for closed extensions of adjoint pairs of operators with applications to elliptic boundary value problems, Math. Nachr., 3 (2009), 314 - 347.

[13] B. M. Brown, J. Hinchcliffe, M. Marletta, S. Naboko and I. Wood: The abstract Titchmarsh-Weyl $M$-function for adjoint operator pairs and its relation to the spectrum, Int. Eq. Oper. Th, 63 (2009), 297 - 320.

[14] B. M. Brown, M. Marletta, S. Naboko and I. Wood: Boundary triplets and $M$-functions for non-selfadjoint operators, with applications to elliptic PDEs and block operator matrices, J. London Math. Soc., (2) 77 (2008), 700-718.

[15] M. Crandall and R. Phillips: On the extension problem for dissipative operators, Journ. Func. Anal. 2 (1968), 147-176.

[16] V. Derkach and M. Malamud: Generalized resolvents and the boundary value problems for Hermitian operators with gaps, J. Funct. Anal. 95 (1991), 1-95.

[17] D.E. Edmunds and W.D. Evans: Spectral Theory and Differential Operators, Oxford University Press, Oxford, 1987.

[18] A.F.M. ter Elst, M. Sauter and H. Vogt: A generalisation of the form method for accretive forms and operators, J. Funct. Anal. 269 (2015), no. 3, 705-744.

[19] W.D. Evans and I. Knowles: On the Extension Problem for Accretive Differential Operators, Journ. Func. Anal. 63 (1985), no. 3, 276-298.

[20] W.D. Evans and I. Knowles: On the Extension Problem for Singular Accretive Differential Operators, Journ. Diff. Equ. 63 (1986), 264-288.

[21] F. Gesztesy, M. Mitrea and M. Zinchenko: Variations on a theme by Jost and Pais, J. Funct. Anal., 253 (2007) 399-448.

[22] F. Gesztesy and M. Mitrea: Generalized Robin boundary conditions, Robinto-Dirichlet maps, and Krein-type resolvent formulas for Schrödinger operators on bounded Lipschitz domains, In Perspectives in partial differential equations, harmonic analysis and applications, volume 79 of Proc. Sympos. Pure Math., pages 105-173. Amer. Math. Soc., Providence, RI, 2008.

[23] F. Gesztesy and M. Mitrea: Nonlocal Robin Laplacians and some remarks on a paper by Filonov on eigenvalue inequalities, J. Diff. Eq., 247:2871-2896, 2009. 
[24] F. Gesztesy and M. Mitrea: Robin-to-Robin maps and Krein-type resolvent formulas for Schrödinger operators on bounded Lipschitz domains, Oper. Theory Adv. Appl., 191 (2009), 81-113.

[25] F. Gesztesy and M. Mitrea: A description of all selfadjoint extensions of the Laplacian and Krein-type resolvent formulas in nonsmooth domains, J. Analyse Math., 113 (2011), 53-172.

[26] G. Grubb: Krein resolvent formulas for elliptic boundary problems in nonsmooth domains, Rend. Sem. Mat. Univ. Pol. Torino, 66 (2008), 13-39.

[27] G. Grubb: Spectral asymptotics for nonsmooth singular Green operators, arXiv:1205.0094. To appear in Comm. Part. Diff. Eq.

[28] G. Grubb: A characterization of the non-local boundary value problems associated with an elliptic operator, Annali della Scuola Normale Superiore di Pisa, Classe di Scienze $3^{e}$ série, 22 (1968), 425-513.

[29] S. Hassi, M. Malamud and V. Mogilevskii: Unitary Equivalence of Proper Extensions of a Symmetric Operator and the Weyl Function, Integral Equations Operator Theory, 77, 2013, 449-487.

[30] P. Hess, T. Kato: Perturbation of Closed Operators and Their Adjoints, Commentarii Mathematici Helvetici, 45 (1970), 524-529.

[31] T. Kato: Perturbation Theory for Linear Operators, Springer-Verlag, New York, 1966.

[32] M.G.Kreun: The theory of selfadjoint extensions of semibounded Hermitian transformations and its applications, I. Mat. Sbornik 20 (1947), no. 3, 431-495 (Russian).

[33] V.E. Lyantze and O.G. Storozh: Methods of the Theory of Unbounded Operators, Naukova Dumka, Kiev, 1983 (Russian).

[34] M. Malamud: Operator holes and extensions of sectorial operators and dual pairs of contractions, Math. Nach. 279 (2006), 625-655.

[35] M. Malamud and V. Mogilevskii: On extensions of dual pairs of operators, Dopovidi Nation. Akad. Nauk Ukrainy, 1 (1997) 30-37.

[36] M. Malamud and V. Mogilevskii: On Weyl functions and $Q$-function of dual pairs of linear relations, Dopovidi Nation. Akad. Nauk Ukrainy 4 (1999) 32-37.

[37] M. Malamud and V. Mogilevskii: Kreŭn type formula for canonical resolvents of dual pairs of linear relations, Methods Funct. Anal. Topology (4) 8 (2002), $72-100$.

[38] J. von Neumann: Allgemeine Eigenwerttheorie Hermitescher Funktionaloperatoren, Math. Ann. 102 (1929), 49-131 (German).

[39] R. Phillips: Dissipative operators and hyperbolic systems of partial differential equations, Trans. Amer. Math. Soc. 90 (1959), 192-254.

[40] B. Sz.-Nagy, C. Foias, H. Bercovici and L. Kérchy: Harmonic Analysis of Operators on Hilbert Space, Springer Science \& Business Media, 2010.

[41] S. Trostorff: A characterization of boundary conditions yielding maximal monotone operators, J. Funct. Anal. 267 (2014), 2787-2822.

[42] M.I. Vishik: On general boundary conditions for elliptic differential equations, Tr. Mosk. Mat. Obs., 1 (1952), 187-246 (Russian); Amer. Math. Soc. Trans. 24 (1963), 107-172. 
[43] J. Weidmann: Linear Operators in Hilbert Spaces, Springer-Verlag, New York, 1980.

Christoph Fischbacher

School of Mathematics, Statistics and Actuarial Science

University of Kent

Canterbury, Kent CT2 7NF

United Kingdom

e-mail: cf299@kent.ac.uk

Sergey Naboko

Department of Math. Physics

Institute of Physics

St. Petersburg State University 1 Ulianovskaia

St. Petergof, St. Petersburg 198504

Russia

e-mail: sergey.naboko@gmail.com

Ian Wood

School of Mathematics, Statistics and Actuarial Science

University of Kent

Canterbury, Kent CT2 7NF

United Kingdom

e-mail: i.wood@kent.ac.uk 